TRANSACTIONS OF THE

AMERICAN MATHEMATICAL SOCIETY

Volume 362, Number 11, November 2010, Pages 5947-5965

S 0002-9947(2010)05172-4

Article electronically published on June 14, 2010

\title{
FUNCTIONAL EQUATIONS OF $L$-FUNCTIONS FOR SYMMETRIC PRODUCTS OF THE KLOOSTERMAN SHEAF
}

\author{
LEI FU AND DAQING WAN
}

\begin{abstract}
We determine the (arithmetic) local monodromy at 0 and at $\infty$ of the Kloosterman sheaf using local Fourier transformations and Laumon's stationary phase principle. We then calculate $\epsilon$-factors for symmetric products of the Kloosterman sheaf. Using Laumon's product formula, we get functional equations of $L$-functions for these symmetric products and prove a conjecture of Evans on signs of constants of functional equations.
\end{abstract}

\section{INTRODUCTION}

Let $p \neq 2$ be a prime number and let $\mathbb{F}_{p}$ be the finite field with $p$ elements. Fix an algebraic closure $\mathbb{F}$ of $\mathbb{F}_{p}$. Denote the projective line over $\mathbb{F}_{p}$ by $\mathbb{P}^{1}$. For any power $q$ of $p$, let $\mathbb{F}_{q}$ be the finite subfield of $\mathbb{F}$ with $q$ elements. Let $\ell$ be a prime number different from $p$. Fix a nontrivial additive character $\psi: \mathbb{F}_{p} \rightarrow \overline{\mathbb{Q}}_{\ell}^{*}$. For any $x \in \mathbb{F}_{q}^{*}$, we define the one-variable Kloosterman sum by

$$
\mathrm{Kl}_{2}\left(\mathbb{F}_{q}, x\right)=\sum_{x \in \mathbb{F}_{q}^{*}} \psi\left(\operatorname{Tr}_{\mathbb{F}_{q} / \mathbb{F}_{p}}\left(\lambda+\frac{x}{\lambda}\right)\right) .
$$

In [3], Deligne constructs a lisse $\overline{\mathbb{Q}}_{l}$-sheaf $\mathrm{Kl}_{2}$ of rank 2 on $\mathbb{G}_{m}=\mathbb{P}^{1}-\{0, \infty\}$, which we call the Kloosterman sheaf, such that for any $x \in \mathbb{G}_{m}\left(\mathbb{F}_{q}\right)=\mathbb{F}_{q}^{*}$, we have

$$
\operatorname{Tr}\left(F_{x}, \mathrm{Kl}_{2, \bar{x}}\right)=-\mathrm{Kl}_{2}\left(\mathbb{F}_{q}, x\right),
$$

where $F_{x}$ is the geometric Frobenius element at the point $x$. For a positive integer $k$, the $L$-function $L\left(\mathbb{G}_{m}, \operatorname{Sym}^{k}\left(\mathrm{Kl}_{2}\right), T\right)$ of the $k$-th symmetric product of $\mathrm{Kl}_{2}$ was first studied by Robba [16] via Dwork's $p$-adic methods. Motivated by applications in coding theory, by connections with modular forms, $p$-adic modular forms and Dwork's unit root zeta functions, there has been a great deal of recent interest to understand $L\left(\mathbb{G}_{m}, \operatorname{Sym}^{k}\left(\mathrm{Kl}_{2}\right), T\right)$ as much as possible for all $k$ and for all $p$. This quickly raises a large number of interesting new problems.

Let $j: \mathbb{G}_{m} \rightarrow \mathbb{P}^{1}$ be the inclusion. We shall be interested in the $L$-function

$$
M_{k}(p, T):=L\left(\mathbb{P}^{1}, j_{*}\left(\operatorname{Sym}^{k}\left(\mathrm{Kl}_{2}\right)\right), T\right) .
$$

This is the nontrivial factor of $L\left(\mathbb{G}_{m}, \operatorname{Sym}^{k}\left(\mathrm{Kl}_{2}\right), T\right)$. The trivial factor of $L\left(\mathbb{G}_{m}, \operatorname{Sym}^{k}\left(\mathrm{Kl}_{2}\right), T\right)$ was completely determined in Fu-Wan [7. By the general

Received by the editors January 4, 2009.

2000 Mathematics Subject Classification. Primary 11L05, 14 G15.

Key words and phrases. Kloosterman sheaf, $\epsilon$-factor, $\ell$-adic Fourier transformation.

The research of the first author was supported by the NSFC (10525107). 
theory of Grothendieck-Deligne, the nontrivial factor $M_{k}(p, T)$ is a polynomial in $T$ with integer coefficients, pure of weight $k+1$. Its degree $\delta_{k}(p)$ can be easily extracted from Fu-Wan [8], Proposition 2.3, Lemmas 4.1 and 4.2:

$$
\delta_{k}(p)= \begin{cases}\frac{k-1}{2}-\left[\frac{k}{2 p}+\frac{1}{2}\right] & \text { if } k \text { is odd } \\ 2\left(\left[\frac{k-2}{4}\right]-\left[\frac{k}{2 p}\right]\right) & \text { if } k \text { is even. }\end{cases}
$$

For fixed $k$, the variation of $M_{k}(p, T)$ as $p$ varies should be explained by an automorphic form; see Choi-Evans [2] and Evans [4 for the precise relations in the cases $k \leq 7$ and Fu-Wan 9] for a motivic interpretation for all $k$. For $k \leq 4$, the degree $\delta_{k}(p) \leq 1$ and $M_{k}(p, T)$ can be determined easily. For $k=5$, the degree $\delta_{5}(p)=2$ for $p>5$. The quadratic polynomial $M_{5}(p, T)$ is explained by an explicit modular form [15]. For $k=6$, the degree $\delta_{6}(p)=2$ for $p>6$. The quadratic polynomial $M_{6}(p, T)$ is again explained by an explicit modular form [10]. For $k=7$, the degree $\delta_{7}(p)=3$ for $p>7$. The cubic polynomial $M_{7}(p, T)$ is conjecturally explained in a more subtle way by an explicit modular form in Evans 4. We will return to this conjecture later in the Introduction.

For fixed $p$, the variation of $M_{k}(p, T)$ as $k$ varies $p$-adically should be related to $p$-adic automorphic forms and $p$-adic $L$-functions. No progress has been made along this direction. The $p$-adic limit of $M_{k}(p, T)$ as $k$ varies $p$-adically links to an important example of Dwork's unit root zeta function; see the introduction in Wan [19. The polynomial $M_{k}(p, T)$ can be used to determine the weight distribution of certain codes, see Moisio [13, 14, and this has been studied extensively for small $p$ and small $k$. The $p$-adic Newton polygon (the $p$-adic slopes) of $M_{k}(p, T)$ remains largely mysterious.

By Katz [1], 4.1.11, we have $\left(\mathrm{Kl}_{2}\right)^{\vee}=\mathrm{Kl}_{2} \otimes \overline{\mathbb{Q}}_{\ell}(1)$. So for any natural number $k$, we have

$$
\left(\operatorname{Sym}^{k}\left(\mathrm{Kl}_{2}\right)\right)^{\vee}=\operatorname{Sym}^{k}\left(\mathrm{Kl}_{2}\right) \otimes \overline{\mathbb{Q}}_{\ell}(k) .
$$

The general theory (confer [12, 3.1.1) shows that $M_{k}(p, T)$ satisfies the functional equation

$$
M_{k}(p, T)=c T^{\delta} M_{k}\left(p, \frac{1}{p^{k+1} T}\right)
$$

where

$$
\begin{aligned}
c & =\prod_{i=0}^{2} \operatorname{det}\left(-F, H^{i}\left(\mathbb{P}_{\mathbb{F}}^{1}, j_{*}\left(\operatorname{Sym}^{k}\left(\mathrm{Kl}_{2}\right)\right)\right)\right)^{(-1)^{i+1}}, \\
\delta & =-\chi\left(\mathbb{P}_{\mathbb{F}}^{1}, j_{*}\left(\operatorname{Sym}^{k}\left(\mathrm{Kl}_{2}\right)\right)\right)=\delta_{k}(p),
\end{aligned}
$$

and $F$ denotes the Frobenius correspondence. Applying the functional equation twice, we get

$$
c^{2}=p^{(k+1) \delta} \text {. }
$$

Based on numerical computation, Evans (44, 5]) suggests that the sign of $c$ should be $-\left(\frac{p}{105}\right)$ (the Jacobi symbol) for $k=7,-\left(\frac{p}{1155}\right)$ for $k=11$, and $\left(\frac{p}{15015}\right)$ for $k=13$. In this paper, we determine $c$ for all $k$ and all $p>2$. The main result of this paper is the following theorem.

Theorem 0.1. Let $p>2$ be an odd prime. If $k$ is even, we have

$$
c=p^{(k+1)\left(\left[\frac{k-2}{4}\right]-\left[\frac{k}{2 p}\right]\right)} .
$$


If $k$ is odd, we have

$$
c=(-1)^{\frac{k-1}{2}+\left[\frac{k}{2 p}+\frac{1}{2}\right]} p^{\frac{k+1}{2}\left(\frac{k-1}{2}-\left[\frac{k}{2 p}+\frac{1}{2}\right]\right)}\left(\frac{-2}{p}\right)^{\left[\frac{k}{2 p}+\frac{1}{2}\right]} \prod_{\substack{j \in\left\{0,1, \ldots,\left[\frac{k}{2}\right]\right\}, p \nmid 2 j+1}}\left(\frac{(-1)^{j}(2 j+1)}{p}\right) .
$$

Corollary 0.2. If $k$ is even and $p>2$, the sign of $c$ is always 1 . If $k$ is odd and $p>k$, the sign of $c$ is

$$
(-1)^{\frac{k-1}{2}} \prod_{j \in\left\{0,1, \ldots,\left[\frac{k}{2}\right]\right\}, p \nmid 2 j+1}\left(\frac{(-1)^{j}(2 j+1)}{p}\right) .
$$

In the above corollary, if we take $k=7$, we see that the sign of $c$ for $p>7$ is

$$
-\left(\frac{1 \cdot(-3) \cdot 5 \cdot(-7)}{p}\right)=-\left(\frac{105}{p}\right)=-\left(\frac{p}{105}\right) ;
$$

if we take $k=11$, we see that the sign of $c$ for $p>11$ is

$$
-\left(\frac{1 \cdot(-3) \cdot 5 \cdot(-7) \cdot 9 \cdot(-11)}{p}\right)=-\left(\frac{-1155}{p}\right)=-\left(\frac{p}{1155}\right)
$$

if we take $k=13$, we see that the $\operatorname{sign}$ of $c$ for $p>13$ is

$$
\left(\frac{1 \cdot(-3) \cdot 5 \cdot(-7) \cdot 9 \cdot(-11) \cdot 13}{p}\right)=\left(\frac{-15015}{p}\right)=\left(\frac{p}{15015}\right)
$$

consistent with Evans' calculation.

In the case $k=7$, Evans proposed a precise description of $M_{7}(p, T)$ in terms of modular forms. For $k=7$ and $p>7$, the polynomial $M_{7}(p, T)$ has degree 3 . Write

$$
M_{7}(p, T)=1+a_{p} T+d_{p} T^{2}+e_{p} T^{3} .
$$

The functional equation and our sign determination show that one of the reciprocal roots for $M_{7}(p, T)$ is $\left(\frac{p}{105}\right) p^{4}$ and $e_{p}=-\left(\frac{p}{105}\right) p^{12}$. Denote the other two reciprocal roots by $\lambda_{p}$ and $\mu_{p}$, which are Weil numbers of weight 8 . We deduce that

$$
a_{p}=-\left(\left(\frac{p}{105}\right) p^{4}+\lambda_{p}+\mu_{p}\right), \lambda_{p} \mu_{p}=p^{8},\left|\lambda_{p}\right|=\left|\mu_{p}\right|=p^{4} .
$$

To explain the numerical calculation of Evans, Katz suggests that there exists a two-dimensional representation

$$
\rho: \operatorname{Gal}(\overline{\mathbb{Q}} / \mathbb{Q}) \rightarrow \mathrm{GL}\left(\overline{\mathbb{Q}}_{\ell}^{2}\right)
$$

unramified for $p>7$ and a Dirichlet character $\chi$ such that

$$
\begin{aligned}
\alpha_{p}^{2} & =\chi(p)\left(\frac{p}{105}\right) \frac{\lambda_{p}}{p^{4}}, \\
\beta_{p}^{2} & =\chi(p)\left(\frac{p}{105}\right) \frac{\mu_{p}}{p^{4}}, \\
\alpha_{p} \beta_{p} & =\chi(p),
\end{aligned}
$$

where $\alpha_{p}$ and $\beta_{p}$ are the eigenvalues of the geometric Frobenius element at $p$ under $\rho$. We then have

$$
\begin{aligned}
1-\left(\frac{p}{105}\right) \frac{a_{p}}{p^{4}} & =2+\left(\frac{p}{105}\right) \frac{\lambda_{p}}{p^{4}}+\left(\frac{p}{105}\right) \frac{\mu_{p}}{p^{4}} \\
& =\bar{\chi}(p)\left(2 \alpha_{p} \beta_{p}+\alpha_{p}^{2}+\beta_{p}^{2}\right) \\
& =\bar{\chi}(p)\left(\alpha_{p}+\beta_{p}\right)^{2} .
\end{aligned}
$$


Set $b(p)=p\left(\alpha_{p}+\beta_{p}\right)$. Evans [4] conjectured that $b(p)$ is the $p$-th Hecke eigenvalue for a weight 3 newform $f$ on $\Gamma_{0}(525)$. Our $a_{p}$ equals $-c_{p} p^{2}$ in 4 .

Our proof of Theorem 0.1 naturally splits into two parts, corresponding to the two ramification points at 0 and $\infty$. Let $t$ be the coordinate of $\mathbb{A}^{1}=\mathbb{P}^{1}-\{\infty\}$. For any closed point $x$ in $\mathbb{P}^{1}$, let $\mathbb{P}_{(x)}^{1}$ be the henselization of $\mathbb{P}^{1}$ at $x$. By Laumon's product formula [12], 3.2.1.1, we have

$$
c=p^{k+1} \prod_{x \in\left|\mathbb{P}^{1}\right|} \epsilon\left(\mathbb{P}_{(x)}^{1},\left.j_{*}\left(\operatorname{Sym}^{k}\left(\mathrm{Kl}_{2}\right)\right)\right|_{\mathbb{P}_{(x)}^{1}},\left.d t\right|_{\left.\mathbb{P}_{(x)}^{1}\right)},\right.
$$

where $\left|\mathbb{P}^{1}\right|$ is the set of all closed points of $\mathbb{P}^{1}$. When $x \neq 0, \infty$, the sheaf $\left.\operatorname{Sym}^{k}\left(\mathrm{Kl}_{2}\right)\right|_{\mathbb{P}_{(x)}^{1}}$ is lisse and the order of $d t$ at $x$ is 0 . So by [12, 3.1.5.4 (ii) and (v), we have

$$
\epsilon\left(\mathbb{P}_{(x)}^{1},\left.j_{*}\left(\operatorname{Sym}^{k}\left(\mathrm{Kl}_{2}\right)\right)\right|_{\mathbb{P}_{(x)}^{1}},\left.d t\right|_{\mathbb{P}_{(x)}^{1}}\right)=1
$$

for $x \neq 0, \infty$. Therefore

$$
c=p^{k+1} \epsilon\left(\mathbb{P}_{(0)}^{1},\left.j_{*}\left(\operatorname{Sym}^{k}\left(\mathrm{Kl}_{2}\right)\right)\right|_{\mathbb{P}_{(0)}^{1}},\left.d t\right|_{\mathbb{P}_{(0)}^{1}}\right) \epsilon\left(\mathbb{P}_{(\infty)}^{1},\left.j_{*}\left(\operatorname{Sym}^{k}\left(\mathrm{Kl}_{2}\right)\right)\right|_{\mathbb{P}_{(\infty)}^{1}},\left.d t\right|_{\left.\mathbb{P}_{(\infty)}^{1}\right)}\right) .
$$

In $\S 1$, we prove the following.

Proposition 0.3. We have

$$
\epsilon\left(\mathbb{P}_{(0)}^{1},\left.j_{*}\left(\operatorname{Sym}^{k}\left(\mathrm{Kl}_{2}\right)\right)\right|_{\mathbb{P}_{(0)}^{1}},\left.d t\right|_{\mathbb{P}_{(0)}^{1}}\right)=(-1)^{k} p^{\frac{k(k+1)}{2}} .
$$

In $\S 2$, we prove the following.

Proposition 0.4. $\epsilon\left(\mathbb{P}_{(\infty)}^{1},\left.j_{*}\left(\operatorname{Sym}^{k}\left(\mathrm{Kl}_{2}\right)\right)\right|_{\left.\mathbb{P}_{(\infty)}^{1}\right)},\left.d t\right|_{\left.\mathbb{P}_{(\infty)}^{1}\right)}\right)$ equals

$$
p^{-(k+1)\left(\frac{k+8}{4}+\left[\frac{k}{2 p}\right]\right)}
$$

if $k=2 r$ for an even $r$,

$$
p^{-(k+1)\left(\frac{k+6}{4}+\left[\frac{k}{2 p}\right]\right)}
$$

if $k=2 r$ for an odd $r$, and

$$
(-1)^{\frac{k+1}{2}+\left[\frac{k}{p}\right]-\left[\frac{k}{2 p}\right]} p^{-\frac{k+1}{2}\left(\frac{k+5}{2}+\left[\frac{k}{p}\right]-\left[\frac{k}{2 p}\right]\right)}\left(\frac{-2}{p}\right)^{\left[\frac{k}{p}\right]-\left[\frac{k}{2 p}\right]} \prod_{\substack{j \in\left\{0,1, \ldots,\left[\frac{k}{2}\right]\right\}, p \nmid 2 j+1}}\left(\frac{(-1)^{j}(2 j+1)}{p}\right)
$$

if $k=2 r+1$.

We deduce from the above two propositions the constant $c$ as stated in Theorem 0.1 using the following facts:

$$
\begin{aligned}
& {\left[\frac{k-2}{4}\right]= \begin{cases}\frac{k-4}{4} & \text { if } k=2 r \text { for an even } r, \\
\frac{k-2}{4} & \text { if } k=2 r \text { for an odd } r,\end{cases} } \\
& {\left[\frac{k}{p}\right]-\left[\frac{k}{2 p}\right]=\left[\frac{k}{2 p}+\frac{1}{2}\right] \text { if } k \text { is odd. }}
\end{aligned}
$$

To get Proposition 0.4, we first have to determine the local (arithmetic) monodromy of $\mathrm{Kl}_{2}$ at $\infty$. This is Theorem 2.1 in $\S 2$, which is of interest itself, and is proved by using local Fourier transformations and Laumon's stationary phase principle. 


$$
\text { 1. Calculation of } \epsilon\left(\mathbb{P}_{(0)}^{1},\left.j_{*}\left(\operatorname{Sym}^{k}\left(\mathrm{Kl}_{2}\right)\right)\right|_{\mathbb{P}_{(0)}^{1}},\left.d t\right|_{\mathbb{P}_{(0)}^{1}}\right)
$$

Let $\eta_{0}$ be the generic point of $\mathbb{P}_{(0)}^{1}$, let $\bar{\eta}_{0}$ be a geometric point located at $\eta_{0}$, and let $V$ be a $\overline{\mathbb{Q}}_{\ell}$-representation of $\operatorname{Gal}\left(\bar{\eta}_{0} / \eta_{0}\right)$. Suppose the inertia subgroup $I_{0}$ of $\operatorname{Gal}\left(\bar{\eta}_{0} / \eta_{0}\right)$ acts unipotently on $V$. Consider the $\ell$-adic part of the cyclotomic character

$$
t_{\ell}: I_{0} \rightarrow \mathbb{Z}_{\ell}(1), \sigma \mapsto\left(\frac{\sigma\left(\sqrt[\ell^{n}]{t}\right)}{\sqrt[\ell^{n}]{t}}\right)
$$

Note that for any $\sigma$ in the inertia subgroup, the $\ell^{n}$-th root of unity $\frac{\sigma(\sqrt[\ell]{t})}{\ell^{n}}$ does not depend on the choice of the $\ell^{n}$-th root $\sqrt[\ell^{n}]{t}$ of $t$. Since $I_{0}$ acts on $V$ unipotently, there exists a nilpotent homomorphism

$$
N: V(1) \rightarrow V
$$

such that the action of $\sigma \in I_{0}$ on $V$ is given by $\exp \left(t_{\ell}(\sigma)\right.$. $\left.N\right)$. Fix a lifting $F \in$ $\operatorname{Gal}\left(\bar{\eta}_{0} / \eta_{0}\right)$ of the geometric Frobenius element in $\operatorname{Gal}\left(\mathbb{F} / \mathbb{F}_{p}\right)$.

Lemma 1.1. Notation as above. Let $V=\mathrm{Kl}_{2, \bar{\eta}_{0}}$. There exists a basis $\left\{e_{0}, e_{1}\right\}$ of $V$ such that

$$
\begin{aligned}
& F\left(e_{0}\right)=e_{0}, F\left(e_{1}\right)=p e_{1}, \\
& N\left(e_{0}\right)=0, N\left(e_{1}\right)=e_{0} .
\end{aligned}
$$

Proof. This is the $n=2$ case of Proposition 1.1 in [7].

Lemma 1.2. Keep the notation in Lemma 1.1. Let $\left\{f_{0}, \ldots, f_{k}\right\}$ be the basis of $\operatorname{Sym}^{k}(V)=\operatorname{Sym}^{k}\left(\mathrm{Kl}_{2, \bar{\eta}_{0}}\right)$ defined by $f_{i}=\frac{1}{i !} e_{0}^{k-i} e_{1}^{i}$. We have

$$
F\left(f_{i}\right)=p^{i} f_{i}, \quad N\left(f_{i}\right)=f_{i-1},
$$

where we regard $f_{i-1}$ as 0 if $i=0$.

Proof. Use the fact that for any $v_{1}, \ldots, v_{k} \in V$, we have the following identities in $\operatorname{Sym}^{k}(V)$ :

$$
\begin{aligned}
F\left(v_{1} \cdots v_{k}\right) & =F\left(v_{1}\right) \cdots F\left(v_{k}\right) \\
N\left(v_{1} \cdots v_{k}\right) & =\sum_{i=1}^{k} v_{1} \cdots v_{i-1} N\left(v_{i}\right) v_{i+1} \cdots v_{k} .
\end{aligned}
$$

Corollary 1.3. The sheaf $\left.\mathrm{Sym}^{k}\left(\mathrm{Kl}_{2}\right)\right|_{\eta_{0}}$ has a filtration

$$
0=\mathcal{F}_{-1} \subset \mathcal{F}_{0} \subset \cdots \subset \mathcal{F}_{k}=\left.\operatorname{Sym}^{k}\left(\mathrm{Kl}_{2}\right)\right|_{\eta_{0}}
$$

such that

$$
\mathcal{F}_{i} / \mathcal{F}_{i-1} \cong \overline{\mathbb{Q}}_{l}(-i)
$$

for any $i=0, \ldots, k$.

Proof. This follows from Lemma 1.2 by taking $\mathcal{F}_{i}$ to be the sheaf on $\eta_{0}$ corresponding to the Galois representation $\operatorname{Span}\left(f_{0}, \ldots, f_{i}\right)$ of $\operatorname{Gal}\left(\bar{\eta}_{0} / \eta_{0}\right)$.

The following is Proposition 0.3 in the Introduction. 
Proposition 1.4. We have

$$
\epsilon\left(\mathbb{P}_{(0)}^{1},\left.j_{*}\left(\operatorname{Sym}^{k}\left(\mathrm{Kl}_{2}\right)\right)\right|_{\mathbb{P}_{(0)}^{1}},\left.d t\right|_{\mathbb{P}_{(0)}^{1}}\right)=(-1)^{k} p^{\frac{k(k+1)}{2}} .
$$

Proof. Let $u: \eta_{0} \rightarrow \mathbb{P}_{(0)}^{1}$ and $v:\{0\} \rightarrow \mathbb{P}_{(0)}^{1}$ be the immersions. By [12] 3.1.5.4 (iii) and (v), we have

$$
\begin{aligned}
& \epsilon\left(\mathbb{P}_{(0)}^{1}, u_{*} \overline{\mathbb{Q}}_{\ell}(-i),\left.d t\right|_{\mathbb{P}_{(0)}^{1}}\right)=1, \\
& \epsilon\left(\mathbb{P}_{(0)}^{1}, v_{*} \overline{\mathbb{Q}}_{\ell}(-i),\left.d t\right|_{\mathbb{P}_{(0)}^{1}}\right)=\operatorname{det}\left(-F_{0}, \overline{\mathbb{Q}}_{\ell}(-i)\right)^{-1}=-\frac{1}{p^{i}} .
\end{aligned}
$$

We have an exact sequence

$$
0 \rightarrow u_{!} \overline{\mathbb{Q}}_{\ell}(-i) \rightarrow u_{*} \overline{\mathbb{Q}}_{\ell}(-i) \rightarrow v_{*} \overline{\mathbb{Q}}_{\ell}(-i) \rightarrow 0 .
$$

It follows from [12, 3.1.5.4 (ii) that we have

$$
\begin{aligned}
\epsilon\left(\mathbb{P}_{(0)}^{1}, u_{!} \overline{\mathbb{Q}}_{\ell}(-i),\left.d t\right|_{\mathbb{P}_{(0)}^{1}}\right) & =\frac{\epsilon\left(\mathbb{P}_{(0)}^{1}, u_{*} \overline{\mathbb{Q}}_{\ell}(-i),\left.d t\right|_{\mathbb{P}_{(0)}^{1}}\right)}{\epsilon\left(\mathbb{P}_{(0)}^{1}, v_{*} \overline{\mathbb{Q}}_{\ell}(-i),\left.d t\right|_{\mathbb{P}_{(0)}^{1}}\right)} \\
& =-p^{i} .
\end{aligned}
$$

By Corollary 1.3 and [12, 3.1.5.4 (ii), we have

$$
\begin{aligned}
\epsilon\left(\mathbb{P}_{(0)}^{1},\left.j_{!}\left(\operatorname{Sym}^{k}\left(\mathrm{Kl}_{2}\right)\right)\right|_{\mathbb{P}_{(0)}^{1}},\left.d t\right|_{\mathbb{P}_{(0)}^{1}}\right) & =\prod_{i=0}^{k} \epsilon\left(\mathbb{P}_{(0)}^{1}, u_{!}\left(\mathcal{F}_{i} / \mathcal{F}_{i+1}\right),\left.d t\right|_{\mathbb{P}_{(0)}^{1}}\right) \\
& =\prod_{i=0}^{k} \epsilon\left(\mathbb{P}_{(0)}^{1}, u_{!} \mathbb{Q}_{\ell}(-i),\left.d t\right|_{\mathbb{P}_{(0)}^{1}}\right) \\
& =\prod_{i=0}^{k}\left(-p^{i}\right) .
\end{aligned}
$$

Moreover, by Lemma 1.2, we have

$$
v^{*}\left(\left.j_{*}\left(\operatorname{Sym}^{k}\left(\mathrm{Kl}_{2}\right)\right)\right|_{\mathbb{P}_{(0)}^{1}}\right) \cong \overline{\mathbb{Q}}_{\ell},
$$

and hence

$$
\epsilon\left(\mathbb{P}_{(0)}^{1}, v_{*} v^{*}\left(\left.j_{*}\left(\operatorname{Sym}^{k}\left(\mathrm{Kl}_{2}\right)\right)\right|_{\mathbb{P}_{(0)}^{1}}\right),\left.d t\right|_{\mathbb{P}_{(0)}^{1}}\right)=-1
$$

So we have

$$
\begin{aligned}
& \epsilon\left(\mathbb{P}_{(0)}^{1},\left.j_{*}\left(\operatorname{Sym}^{k}\left(\mathrm{Kl}_{2}\right)\right)\right|_{\mathbb{P}_{(0)}^{1}},\left.d t\right|_{\mathbb{P}_{(0)}^{1}}\right) \\
= & \epsilon\left(\mathbb{P}_{(0)}^{1},\left.j_{!}\left(\operatorname{Sym}^{k}\left(\mathrm{Kl}_{2}\right)\right)\right|_{\mathbb{P}_{(0)}^{1}},\left.d t\right|_{\mathbb{P}_{(0)}^{1}}\right) \epsilon\left(\mathbb{P}_{(0)}^{1}, v_{*} v^{*}\left(\left.j_{*}\left(\operatorname{Sym}^{k}\left(\mathrm{Kl}_{2}\right)\right)\right|_{\left.\mathbb{P}_{(0)}^{1}\right)}\right),\left.d t\right|_{\mathbb{P}_{(0)}^{1}}\right) \\
= & \prod_{i=1}^{k}\left(-p^{i}\right) \\
= & (-1)^{k} p^{\frac{k(k+1)}{2}} .
\end{aligned}
$$


2. Calculation of $\epsilon\left(\mathbb{P}_{(\infty)}^{1},\left.j_{*}\left(\operatorname{Sym}^{k}\left(\mathrm{Kl}_{2}\right)\right)\right|_{\mathbb{P}_{(\infty)}^{1}},\left.d t\right|_{\mathbb{P}_{(\infty)}^{1}}\right)$

We first introduce some notation. Fix a nontrivial additive character $\psi: \mathbb{F}_{p} \rightarrow$ $\overline{\mathbb{Q}}_{\ell}^{*}$ and define $\mathrm{Kl}_{2}$ as in the Introduction. Fix a separable closure $\overline{\mathbb{F}_{p}(t)}$ of $\mathbb{F}_{p}(t)$. Let $x$ be an element in $\overline{\mathbb{F}_{p}(t)}$ satisfying $x^{p}-x=t$. Then $\mathbb{F}_{p}(t, x)$ is Galois over $\mathbb{F}_{p}(t)$. We have a canonical isomorphism

$$
\mathbb{F}_{p} \cong \operatorname{Gal}\left(\mathbb{F}_{p}(t, x) / \mathbb{F}_{p}(t)\right)
$$

which sends each $a \in \mathbb{F}_{p}$ to the element in $\operatorname{Gal}\left(\mathbb{F}_{p}(t, x) / \mathbb{F}_{p}(t)\right)$ defined by $x \mapsto x+a$. Let $\mathcal{L}_{\psi}$ be the Galois representation defined by

$$
\operatorname{Gal}\left(\overline{\mathbb{F}_{p}(t)} / \mathbb{F}_{p}(t)\right) \rightarrow \operatorname{Gal}\left(\mathbb{F}_{p}(t, x) / \mathbb{F}_{p}(t)\right) \stackrel{\cong}{\rightarrow} \mathbb{F}_{p} \stackrel{\psi^{-1}}{\rightarrow} \overline{\mathbb{Q}}_{\ell}^{*}
$$

It is unramified outside $\infty$ and totally wild at $\infty$ with Swan conductor 1 . This Galois representation defines a lisse $\overline{\mathbb{Q}}_{\ell}$-sheaf on $\mathbb{A}^{1}$, which we still denote by $\mathcal{L}_{\psi}$. Let $X$

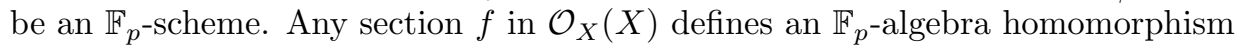

$$
\mathbb{F}_{p}[t] \rightarrow \mathcal{O}_{X}(X), t \mapsto f
$$

and hence an $\mathbb{F}_{p}$-morphism of schemes

$$
f: X \rightarrow \mathbb{A}^{1}
$$

We denote the lisse $\overline{\mathbb{Q}}_{\ell}$-sheaf $f^{*} \mathcal{L}_{\psi}$ on $X$ by $\mathcal{L}_{\psi}(f)$. For any $f_{1}, f_{2} \in \mathcal{O}_{X}(X)$, we have

$$
\mathcal{L}_{\psi}\left(f_{1}\right) \otimes \mathcal{L}_{\psi}\left(f_{2}\right) \cong \mathcal{L}_{\psi}\left(f_{1}+f_{2}\right)
$$

Recall that $p \neq 2$. Let $y$ be an element in $\overline{\mathbb{F}_{p}(t)}$ satisfying $y^{2}=t$. Then $\mathbb{F}_{p}(t, y)$ is Galois over $\mathbb{F}_{p}(t)$. We have a canonical isomorphism

$$
\{ \pm 1\} \stackrel{\cong}{\rightrightarrows} \mathrm{Gal}\left(\mathbb{F}_{p}(t, y) / \mathbb{F}_{p}(t)\right)
$$

which sends -1 to the element in $\operatorname{Gal}\left(\mathbb{F}_{p}(t, y) / \mathbb{F}_{p}(t)\right)$ defined by $y \mapsto-y$. Let

$$
\chi:\{ \pm 1\} \rightarrow \overline{\mathbb{Q}}_{\ell}^{*}
$$

be the (unique) nontrivial character. Define $\mathcal{L}_{\chi}$ to be the Galois representation defined by

$$
\operatorname{Gal}\left(\overline{\mathbb{F}_{p}(t)} / \mathbb{F}_{p}(t)\right) \rightarrow \operatorname{Gal}\left(\mathbb{F}_{p}(t, y) / \mathbb{F}_{p}(t)\right) \stackrel{\cong}{\rightarrow}\{ \pm 1\} \stackrel{\chi^{-1}}{\rightarrow} \overline{\mathbb{Q}}_{\ell}^{*}
$$

It is unramified outside 0 and $\infty$ and tamely ramified at 0 and $\infty$. This Galois representation defines a lisse $\overline{\mathbb{Q}}_{\ell}$-sheaf on $\mathbb{G}_{m}$, which we still denote by $\mathcal{L}_{\chi}$.

Let $\theta: \operatorname{Gal}\left(\mathbb{F} / \mathbb{F}_{p}\right) \rightarrow \overline{\mathbb{Q}}_{\ell}^{*}$ be a character of the Galois group of the finite field. Denote by $\mathcal{L}_{\theta}$ the Galois representation

$$
\operatorname{Gal}\left(\overline{\mathbb{F}_{p}(t)} / \mathbb{F}_{p}(t)\right) \rightarrow \operatorname{Gal}\left(\mathbb{F} / \mathbb{F}_{p}\right) \stackrel{\theta}{\rightarrow} \overline{\mathbb{Q}}_{\ell}^{*}
$$

It is unramified everywhere and hence defines a lisse $\overline{\mathbb{Q}}_{l}$-sheaf on $\mathbb{P}^{1}$, which we still denote by $\mathcal{L}_{\theta}$.

Theorem 2.1. Notation as above. Let $\eta_{\infty}$ be the generic point of $\mathbb{P}_{(\infty)}^{1}$. Then $\left.\mathrm{Kl}_{2}\right|_{\eta_{\infty}}$ is isomorphic to the restriction to $\eta_{\infty}$ of the sheaf

$$
[2]_{*}\left(\mathcal{L}_{\psi}(2 t) \otimes \mathcal{L}_{\chi}\right) \otimes \mathcal{L}_{\theta_{0}}
$$

where $[2]: \mathbb{G}_{m} \rightarrow \mathbb{G}_{m}$ is the morphism defined by $x \mapsto x^{2}$, and

$$
\theta_{0}: \operatorname{Gal}\left(\mathbb{F} / \mathbb{F}_{p}\right) \rightarrow \overline{\mathbb{Q}}_{\ell}^{*}
$$


is the character sending the geometric Frobenius element $F$ in $\operatorname{Gal}\left(\mathbb{F} / \mathbb{F}_{p}\right)$ to the Gauss sum

$$
\theta_{0}(F)=g(\chi, \psi)=-\sum_{x \in \mathbb{F}_{p}^{*}}\left(\frac{x}{p}\right) \psi(x) .
$$

Proof. By [9], Proposition 1.1, we have

$$
\mathrm{Kl}_{2}=\left.\mathcal{F}\left(j ! \mathcal{L}_{\psi}\left(\frac{1}{t}\right)\right)\right|_{\mathbb{G}_{m}},
$$

where $\mathcal{F}$ is the $\ell$-adic Fourier transformation and $j: \mathbb{G}_{m} \rightarrow \mathbb{A}^{1}$ is the inclusion. Let

$$
\pi_{1}, \pi_{2}: \mathbb{G}_{m} \times_{\mathbb{F}_{p}} \mathbb{G}_{m} \rightarrow \mathbb{G}_{m}
$$

be the projections. Using the proper base change theorem and the projection formula ([1], XVII, 5.2.6 and 5.2.9), one can verify

$$
[2]^{*}\left(\left.\mathcal{F}\left(j_{!} \mathcal{L}_{\psi}\left(\frac{1}{t}\right)\right)\right|_{\mathbb{G}_{m}}\right) \cong R \pi_{2 !}\left(\mathcal{L}_{\psi}\left(\frac{1}{t}+t t^{\prime 2}\right)\right)[1],
$$

where

$$
\frac{1}{t}+t t^{\prime 2}: \mathbb{G}_{m} \times_{\mathbb{F}_{p}} \mathbb{G}_{m} \rightarrow \mathbb{A}^{1}
$$

is the morphism corresponding to the $\mathbb{F}_{p}$-algebra homomorphism

$$
\mathbb{F}_{p}[t] \rightarrow \mathbb{F}_{p}\left[t, 1 / t, t^{\prime}, 1 / t^{\prime}\right], t \mapsto \frac{1}{t}+t t^{\prime 2} .
$$

Consider the isomorphism

$$
\tau: \mathbb{G}_{m} \times_{\mathbb{F}_{p}} \mathbb{G}_{m} \rightarrow \mathbb{G}_{m} \times_{\mathbb{F}_{p}} \mathbb{G}_{m},\left(t, t^{\prime}\right) \mapsto\left(\frac{t}{t^{\prime}}, t^{\prime}\right) .
$$

We have $\pi_{2} \tau=\pi_{2}$. So

$$
R \pi_{2 !}\left(\mathcal{L}_{\psi}\left(\frac{1}{t}+t t^{\prime 2}\right)\right) \cong R\left(\pi_{2} \tau\right) ! \tau^{*}\left(\mathcal{L}_{\psi}\left(\frac{1}{t}+t t^{\prime 2}\right)\right) \cong R \pi_{2 !} \mathcal{L}_{\psi}\left(\left(\frac{1}{t}+t\right) t^{\prime}\right) .
$$

Consider the morphism

$$
g: \mathbb{G}_{m} \rightarrow \mathbb{A}^{1}, t \mapsto \frac{1}{t}+t .
$$

Again using the proper base change theorem and the projection formula, one can verify

$$
\mathcal{F}\left(R g ! \overline{\mathbb{Q}}_{\ell}\right) \cong R \pi_{2 !} \mathcal{L}_{\psi}\left(\left(\frac{1}{t}+t\right) t^{\prime}\right)[1]
$$

From the isomorphisms (1)-(4), we get

$$
\left.[2]^{*} \mathrm{Kl}_{2} \cong \mathcal{F}\left(R g_{!} \overline{\mathbb{Q}}_{\ell}\right)\right|_{\mathbb{G}_{m}}
$$

By Lemma 2.2 below, the stationary phase principle of Laumon [12], 2.3.3.1 (iii), and [12, 2.5.3.1, as representations of $\operatorname{Gal}\left(\bar{\eta}_{\infty^{\prime}} / \eta_{\infty^{\prime}}\right)$, we have

$$
\begin{aligned}
\mathcal{H}^{0}\left(\mathcal{F}\left(R g ! \overline{\mathbb{Q}}_{\ell}\right)\right)_{\bar{\eta}_{\infty^{\prime}}} \cong \mathcal{F}^{\left(2, \infty^{\prime}\right)}\left(\mathcal{L}_{\chi}\right) \oplus \mathcal{F}^{\left(-2, \infty^{\prime}\right)}\left(\mathcal{L}_{\chi}\right) \\
\cong\left(\mathcal{L}_{\psi}\left(2 t^{\prime}\right) \otimes \mathcal{F}^{\left(0, \infty^{\prime}\right)}\left(\mathcal{L}_{\chi}\right)\right) \oplus\left(\mathcal{L}_{\psi}\left(-2 t^{\prime}\right) \otimes \mathcal{F}^{\left(0, \infty^{\prime}\right)}\left(\mathcal{L}_{\chi}\right)\right) \\
\cong\left(\mathcal{L}_{\psi}\left(2 t^{\prime}\right) \otimes \mathcal{L}_{\chi} \otimes \mathcal{L}_{\theta_{0}}\right) \oplus\left(\mathcal{L}_{\psi}\left(-2 t^{\prime}\right) \otimes \mathcal{L}_{\chi} \otimes \mathcal{L}_{\theta_{0}}\right)
\end{aligned}
$$

Hence

$$
\left.\left.\left.\left([2]^{*} \mathrm{Kl}_{2}\right)\right|_{\eta_{\infty}} \cong\left(\mathcal{L}_{\psi}(2 t) \otimes \mathcal{L}_{\chi} \otimes \mathcal{L}_{\theta_{0}}\right)\right|_{\eta_{\infty}} \oplus\left(\mathcal{L}_{\psi}(-2 t) \otimes \mathcal{L}_{\chi} \otimes \mathcal{L}_{\theta_{0}}\right)\right|_{\eta_{\infty}}
$$


Note that this decomposition of $\left.\left([2]{ }^{*} \mathrm{Kl}_{2}\right)\right|_{\eta_{\infty}}$ is nonisotypical. By [17, Proposition 24 on p. 61, and the fact that $\left.\mathrm{Kl}_{2}\right|_{\eta_{\infty}}$ is irreducible (since its Swan conductor is 1), we have

We have

$$
\left.\left.\mathrm{Kl}_{2}\right|_{\eta_{\infty}} \cong[2]_{*}\left(\mathcal{L}_{\psi}(2 t) \otimes \mathcal{L}_{\chi} \otimes \mathcal{L}_{\theta_{0}}\right)\right|_{\eta_{\infty}}
$$

$$
\begin{aligned}
{[2]_{*}\left(\mathcal{L}_{\psi}(2 t) \otimes \mathcal{L}_{\chi} \otimes \mathcal{L}_{\theta_{0}}\right) } & \cong[2]_{*}\left(\mathcal{L}_{\psi}(2 t) \otimes \mathcal{L}_{\chi} \otimes[2]^{*} \mathcal{L}_{\theta_{0}}\right) \\
& \cong[2]_{*}\left(\mathcal{L}_{\psi}(2 t) \otimes \mathcal{L}_{\chi}\right) \otimes \mathcal{L}_{\theta_{0}}
\end{aligned}
$$

Here we use the fact that $[2]^{*} \mathcal{L}_{\theta_{0}} \cong \mathcal{L}_{\theta_{0}}$. Hence

$$
\left.\left.\mathrm{Kl}_{2}\right|_{\eta_{\infty}} \cong\left([2]_{*}\left(\mathcal{L}_{\psi}(2 t) \otimes \mathcal{L}_{\chi}\right) \otimes \mathcal{L}_{\theta_{0}}\right)\right|_{\eta_{\infty}}
$$

Lemma 2.2. For the morphism

the following holds:

$$
g: \mathbb{G}_{m} \rightarrow \mathbb{A}^{1}, t \mapsto \frac{1}{t}+t
$$

(i) $R g ! \overline{\mathbb{Q}}_{\ell}$ is a $\overline{\mathbb{Q}}_{\ell}$-sheaf on $\mathbb{A}^{1}$ which is lisse outside the rational points 2 and -2 .

(ii) $R g_{!} \overline{\mathbb{Q}}_{\ell}$ is unramified at $\infty$.

(iii) Let $P$ be one of the rational points 2 or -2 , and let $\tilde{\mathbb{A}}_{(P)}^{1}$ be the henselization of $\mathbb{A}^{1}$ at $P$. We have

$$
\left.\left(R g ! \overline{\mathbb{Q}}_{\ell}\right)\right|_{\tilde{\mathbb{A}}_{(P)}^{1}} \cong \overline{\mathbb{Q}}_{\ell} \oplus \mathcal{L}_{\chi, !}
$$

where $\mathcal{L}_{\chi, !}$ denotes the extension by 0 of the Kummer sheaf $\mathcal{L}_{\chi}$ on the generic point of $\tilde{\mathbb{A}}_{(P)}^{1}$ to $\tilde{\mathbb{A}}_{(P)}^{1}$.

Proof. We have

$$
\frac{\partial g}{\partial t}=-\frac{1}{t^{2}}+1
$$

So $\frac{\partial g}{\partial t}$ vanishes at the points $t= \pm 1$. We have

$$
\begin{aligned}
& g( \pm 1)= \pm 2 \\
& \frac{\partial^{2} g}{\partial t^{2}}( \pm 1)= \pm 2 \neq 0
\end{aligned}
$$

It follows that $g$ is tamely ramified above \pm 2 with ramification index 2 , and $g$ is étale elsewhere. Consider the morphism

$$
\bar{g}: \mathbb{P}^{1} \rightarrow \mathbb{P}^{1},\left[t_{0}: t_{1}\right] \mapsto\left[t_{0} t_{1}: t_{0}^{2}+t_{1}^{2}\right] .
$$

We have $\bar{g}^{-1}(\infty)=\{0, \infty\}$. Hence

$$
\bar{g}^{-1}\left(\mathbb{A}^{1}\right)=\mathbb{G}_{m} .
$$

It is clear that

$$
\left.\bar{g}\right|_{\mathbb{G}_{m}}=g .
$$

So $g: \mathbb{G}_{m} \rightarrow \mathbb{A}^{1}$ is a finite morphism of degree 2 . Near 0 , the morphism $\bar{g}$ can be expressed as

$$
t \mapsto \frac{t}{1+t^{2}}
$$

Hence $\bar{g}$ is unramified at 0 . Similarly $\bar{g}$ is also unramified at $\infty$. Our lemma follows from these facts. 
Remark 2.3. The first attempt to determine the monodromy at $\infty$ of the $(n-1)$ variable Kloosterman sheaf $\left.\mathrm{Kl}_{n}\right|_{\eta_{\infty}}$ is done in Fu-Wan [8, Theorem 1.1, where we deduce from Katz [11] that

$$
\left.\left.\mathrm{Kl}_{n}\right|_{\eta_{\infty}} \cong\left([n]_{*}\left(\mathcal{L}_{\psi}(n t) \otimes \mathcal{L}_{\chi^{n-1}}\right) \otimes \mathcal{L}_{\theta} \otimes \overline{\mathbb{Q}}_{\ell}\left(\frac{1-n}{2}\right)\right)\right|_{\eta_{\infty}}
$$

for some character $\theta: \operatorname{Gal}\left(\mathbb{F} / \mathbb{F}_{p}\right) \rightarrow \overline{\mathbb{Q}}_{\ell}^{*}$, and an explicit description of $\theta^{2}$ is given. Using induction on $n$, 9, Proposition 1.1, and adapting the argument in [6] to a nonalgebraically closed ground field, we can get an explicit description of $\theta$. See 6] where the monodromy of the more general hypergeometric sheaf is treated (over an algebraically closed field).

Lemma 2.4. Keep the notation in Theorem 2.1. Let

$$
\theta_{1}: \operatorname{Gal}\left(\mathbb{F} / \mathbb{F}_{p}\right) \rightarrow \overline{\mathbb{Q}}_{\ell}^{*}
$$

be the character defined by

$$
\theta_{1}(\sigma)=\chi\left(\frac{\sigma(\sqrt{-1})}{\sqrt{-1}}\right)
$$

for any $\sigma \in \operatorname{Gal}\left(\mathbb{F} / \mathbb{F}_{p}\right)$. Note that the above expression is independent of the choice of the square root $\sqrt{-1} \in \mathbb{F}$ of -1 .

(i) If $k=2 r$ is even, $\left.\operatorname{Sym}^{k}\left(\mathrm{Kl}_{2}\right)\right|_{\eta_{\infty}}$ is isomorphic to the restriction to $\eta_{\infty}$ of the sheaf

$$
\left(\mathcal{L}_{\chi^{r}} \otimes \mathcal{L}_{\theta_{0}^{2 r} \theta_{1}^{r}}\right) \oplus\left(\bigoplus_{i=0}^{r-1}[2]_{*} \mathcal{L}_{\psi}((4 i-4 r) t) \otimes \mathcal{L}_{\theta_{0}^{2 r} \theta_{1}^{i}}\right) .
$$

(ii) If $k=2 r+1$ is odd, $\left.\operatorname{Sym}^{k}\left(\mathrm{Kl}_{2}\right)\right|_{\eta_{\infty}}$ is isomorphic to the restriction to $\eta_{\infty}$ of the sheaf

$$
\bigoplus_{i=0}^{r}[2]_{*}\left(\mathcal{L}_{\psi}((4 i-4 r-2) t) \otimes \mathcal{L}_{\chi}\right) \otimes \mathcal{L}_{\theta_{0}^{2 r+1}} \theta_{1}^{i+1} .
$$

Proof. By Theorem 2.1, it suffices to calculate the restriction to $\eta_{\infty}$ of $\operatorname{Sym}^{k}\left([2]_{*}\left(\mathcal{L}_{\psi}(2 t) \otimes \mathcal{L}_{\chi}\right)\right)$. Let $y, z, w$ be elements in $\overline{\mathbb{F}_{p}(t)}$ satisfying

$$
y^{2}=t, z^{p}-z=y, w^{2}=y .
$$

Fix a square root $\sqrt{-1}$ of -1 in $\mathbb{F}$. Then $\mathbb{F}_{p}(z, w, \sqrt{-1})$ and $\mathbb{F}_{p}(y)$ are Galois extensions of $\mathbb{F}_{p}(t)$. Let $G=\operatorname{Gal}\left(\mathbb{F}_{p}(z, w, \sqrt{-1}) / \mathbb{F}_{p}(t)\right)$ and $H=\operatorname{Gal}\left(\mathbb{F}_{p}(z, w, \sqrt{-1}) /\right.$ $\left.\mathbb{F}_{p}(y)\right)$. Then $H$ is normal in $G$, and we have the canonical isomorphisms

$$
G / H \stackrel{\cong}{\rightrightarrows} \operatorname{Gal}\left(\mathbb{F}_{p}(y) / \mathbb{F}_{p}(t)\right) \stackrel{\cong}{\rightrightarrows}\{ \pm 1\} .
$$

Consider the case where $\sqrt{-1}$ does not lie in $\mathbb{F}_{p}$. We have an isomorphism

$$
\mathbb{F}_{p} \times\{ \pm 1\} \times\{ \pm 1\} \stackrel{\cong}{\rightrightarrows} H=\operatorname{Gal}\left(\mathbb{F}_{p}(z, w, \sqrt{-1}) / \mathbb{F}_{p}(y)\right),
$$

which maps $\left(a, \mu^{\prime}, \mu^{\prime \prime}\right) \in \mathbb{F}_{p} \times\{ \pm 1\} \times\{ \pm 1\}$ to the element $g_{\left(a, \mu^{\prime}, \mu^{\prime \prime}\right)} \in \operatorname{Gal}\left(\mathbb{F}_{p}(z, w\right.$, $\left.\sqrt{-1}) / \mathbb{F}_{p}(y)\right)$ defined by

$$
g_{\left(a, \mu^{\prime}, \mu^{\prime \prime}\right)}(z)=z+a, g_{\left(a, \mu^{\prime}, \mu^{\prime \prime}\right)}(w)=\mu^{\prime} w, g_{\left(a, \mu^{\prime}, \mu^{\prime \prime}\right)}(\sqrt{-1})=\mu^{\prime \prime} \sqrt{-1} .
$$

(In the case where $\sqrt{-1}$ lies in $\mathbb{F}_{p}$, we have $\mathbb{F}_{p}(z, w, \sqrt{-1})=\mathbb{F}_{p}(z, w)$, and we have an isomorphism

$$
\mathbb{F}_{p} \times\{ \pm 1\} \stackrel{\cong}{\rightrightarrows} H=\operatorname{Gal}\left(\mathbb{F}_{p}(z, w) / \mathbb{F}_{p}(y)\right)
$$


which maps $(a, \mu) \in \mathbb{F}_{p} \times\{ \pm 1\}$ to the element $g_{(a, \mu)} \in \operatorname{Gal}\left(\mathbb{F}_{p}(z, w) / \mathbb{F}_{p}(y)\right)$ defined by

$$
g_{(a, \mu)}(z)=z+a, g_{(a, \mu)}(w)=\mu w .
$$

The following argument works for this case with slight modification. We leave it to the reader to treat this case.) Let $V$ be a one-dimensional $\overline{\mathbb{Q}}_{\ell}$-vector space with a basis $e_{0}$. Define an action of $H$ on $V$ by

$$
g_{\left(a, \mu^{\prime}, \mu^{\prime \prime}\right)}\left(e_{0}\right)=\psi(-2 a) \chi\left(\mu^{\prime-1}\right) e_{0} .
$$

Then $[2]_{*}\left(\mathcal{L}_{\psi}(2 t) \otimes \mathcal{L}_{\chi}\right)$ is just the composition of $\operatorname{Ind}_{H}^{G}(V)$ with the canonical homomorphism

$$
\operatorname{Gal}\left(\overline{\mathbb{F}_{p}(t)} / \mathbb{F}_{p}(t)\right) \rightarrow \operatorname{Gal}\left(\mathbb{F}_{p}(z, w, \sqrt{-1}) / \mathbb{F}_{p}(t)\right)=G .
$$

Let $g$ be the element in $G=\operatorname{Gal}\left(\mathbb{F}_{p}(z, w, \sqrt{-1}) / \mathbb{F}_{p}(t)\right)$ defined by

$$
g(z)=-z, g(w)=\sqrt{-1} w, g(\sqrt{-1})=\sqrt{-1} .
$$

Then the image of $g$ in $G / H$ is a generator of the cyclic group $G / H$. So $G$ is generated by $g_{\left(a, \mu^{\prime}, \mu^{\prime \prime}\right)} \in H\left(\left(a, \mu^{\prime}, \mu^{\prime \prime}\right) \in \mathbb{F}_{p} \times\{ \pm 1\} \times\{ \pm 1\}\right)$ and $g$. The space $\operatorname{Ind}_{H}^{G}(V)$ has a basis $\left\{e_{0}, e_{1}\right\}$ with

$$
\begin{aligned}
g\left(e_{0}\right) & =e_{1} \\
g_{\left(a, \mu^{\prime}, \mu^{\prime \prime}\right)}\left(e_{0}\right) & =\psi(-2 a) \chi\left(\mu^{\prime-1}\right) e_{0}, \\
g_{\left(a, \mu^{\prime}, \mu^{\prime \prime}\right)}\left(e_{1}\right) & =\psi(2 a) \chi\left(\mu^{\prime-1} \mu^{\prime \prime-1}\right) e_{1}, \\
g\left(e_{1}\right) & =g^{2}\left(e_{0}\right)=g_{(0,-1,1)}\left(e_{0}\right)=-e_{0} .
\end{aligned}
$$

Suppose $k=2 r$ is even. $\operatorname{Sym}^{k}\left(\operatorname{Ind}_{H}^{G}(V)\right)$ has a basis

$$
\left\{e_{1}^{k}, g\left(e_{1}^{k}\right), e_{0} e_{1}^{k-1}, g\left(e_{0} e_{1}^{k-1}\right), \ldots, e_{0}^{r-1} e_{1}^{r+1}, g\left(e_{0}^{r-1} e_{1}^{r+1}\right), e_{0}^{r} e_{1}^{r}\right\}
$$

and for each $i=0,1, \ldots, r$, we have

$$
\begin{aligned}
g_{\left(a, \mu^{\prime}, \mu^{\prime \prime}\right)}\left(e_{0}^{i} e_{1}^{k-i}\right) & =\psi(-2 i a) \chi\left(\mu^{\prime-i}\right) \psi(2(k-i) a) \chi\left(\mu^{\prime-(k-i)} \mu^{\prime \prime-(k-i)}\right) e_{0}^{i} e_{1}^{k-i} \\
& =\psi(2(k-2 i) a) \chi\left(\mu^{\prime-k}\right) \chi\left(\mu^{\prime \prime-(k-i)}\right) e_{0}^{i} e_{1}^{k-i} .
\end{aligned}
$$

Using the fact that $k$ is even and $\chi^{2}=1$, we get

$$
g_{\left(a, \mu^{\prime}, \mu^{\prime \prime}\right)}\left(e_{0}^{i} e_{1}^{k-i}\right)=\psi(2(k-2 i) a) \chi\left(\mu^{\prime \prime i}\right) e_{0}^{i} e_{1}^{k-i}
$$

In particular, we have

Moreover, we have

$$
g_{\left(a, \mu^{\prime}, \mu^{\prime \prime}\right)}\left(e_{0}^{r} e_{1}^{r}\right)=\chi\left(\mu^{\prime \prime r}\right) e_{0}^{r} e_{1}^{r}
$$

$$
g\left(e_{0}^{r} e_{1}^{r}\right)=e_{1}^{r}\left(g\left(e_{1}^{r}\right)\right)=(-1)^{r} e_{0}^{r} e_{1}^{r} .
$$

It follows that

$$
\operatorname{Sym}^{k}\left([2]_{*}\left(\mathcal{L}_{\psi}(2 t) \otimes \mathcal{L}_{\chi}\right)\right) \cong\left(\mathcal{L}_{\chi^{r}} \otimes \mathcal{L}_{\theta_{1}^{r}}\right) \oplus\left(\bigoplus_{i=0}^{r-1}[2]_{*}\left(\mathcal{L}_{\psi}(2(2 i-k) t) \otimes \mathcal{L}_{\theta_{1}^{i}}\right)\right) .
$$

We have

$[2]_{*}\left(\mathcal{L}_{\psi}(2(2 i-k) t) \otimes \mathcal{L}_{\theta_{1}^{i}}\right) \cong[2]_{*}\left(\mathcal{L}_{\psi}(2(2 i-k) t) \otimes[2]^{*} \mathcal{L}_{\theta_{1}^{i}}\right) \cong[2]_{*} \mathcal{L}_{\psi}(2(2 i-k) t) \otimes \mathcal{L}_{\theta_{1}^{i}}$.

So we have

$$
\operatorname{Sym}^{k}\left([2]_{*}\left(\mathcal{L}_{\psi}(2 t) \otimes \mathcal{L}_{\chi}\right)\right) \cong\left(\mathcal{L}_{\chi^{r}} \otimes \mathcal{L}_{\theta_{1}^{r}}\right) \oplus\left(\bigoplus_{i=0}^{r-1}[2]_{*} \mathcal{L}_{\psi}(2(2 i-k) t) \otimes \mathcal{L}_{\theta_{1}^{i}}\right) .
$$


Suppose $k=2 r+1$ is odd. $\operatorname{Sym}^{k}\left(\operatorname{Ind}_{H}^{G}(V)\right)$ has a basis

$$
\left\{e_{1}^{k}, g\left(e_{1}^{k}\right), e_{0} e_{1}^{k-1}, g\left(e_{0} e_{1}^{k-1}\right), \ldots, e_{0}^{r} e_{1}^{r+1}, g\left(e_{0}^{r} e_{1}^{r+1}\right)\right\} .
$$

Using the same calculation as above, we get

$$
\operatorname{Sym}^{k}\left([2]_{*}\left(\mathcal{L}_{\psi}(2 t) \otimes \mathcal{L}_{\chi}\right)\right) \cong \bigoplus_{i=0}^{r}[2]_{*}\left(\mathcal{L}_{\psi}(2(2 i-k) t) \otimes \mathcal{L}_{\chi}\right) \otimes \mathcal{L}_{\theta_{1}^{i+1}} .
$$

Lemma 2.4 follows by twisting the above expressions of $\operatorname{Sym}^{k}\left([2]_{*}\left(\mathcal{L}_{\psi}(2 t) \otimes \mathcal{L}_{\chi}\right)\right)$ by $\mathcal{L}_{\theta_{0}^{k}}$.

Lemma 2.5. Assume $a \in \mathbb{F}_{p}$ is nonzero. We have the following identities.

(i) $\epsilon\left(\mathbb{P}_{(\infty)}^{1}, \overline{\mathbb{Q}}_{\ell},\left.d t\right|_{\left.\mathbb{P}_{(\infty)}^{1}\right)}\right)=\frac{1}{p^{2}}$.

(ii) $\epsilon\left(\mathbb{P}_{(\infty)}^{1}, \overline{\mathbb{Q}}_{\ell},\left.d t^{2}\right|_{\mathbb{P}_{(\infty)}^{1}}\right)=\frac{1}{p^{3}}$.

(iii) $\epsilon\left(\mathbb{P}_{(\infty)}^{1},\left.j_{*} \mathcal{L}_{\chi}\right|_{\mathbb{P}_{(\infty)}^{1}},\left.d t\right|_{\mathbb{P}_{(\infty)}^{1}}\right)=-\frac{g(\chi, \psi)}{p^{2}}$.

(iv) $\epsilon\left(\mathbb{P}_{(\infty)}^{1},\left.j_{*} \mathcal{L}_{\chi}\right|_{\mathbb{P}_{(\infty)}^{1}},\left.d t^{2}\right|_{\mathbb{P}_{(\infty)}^{1}}\right)=-\frac{g(\chi, \psi)}{p^{3}}\left(\frac{-2}{p}\right)$.

(v) $\epsilon\left(\mathbb{P}_{(\infty)}^{1},\left.j_{*}\left(\mathcal{L}_{\psi}(a t) \otimes \mathcal{L}_{\chi}\right)\right|_{\mathbb{P}_{(\infty)}^{1}},\left.d t^{2}\right|_{\mathbb{P}_{(\infty)}^{1}}\right)=\frac{1}{p^{2}}\left(\frac{2 a}{p}\right)$.

(vi) $\epsilon\left(\mathbb{P}_{(\infty)}^{1},\left.j_{*} \mathcal{L}_{\psi}(a t)\right|_{\mathbb{P}_{(\infty)}^{1}},\left.d t^{2}\right|_{\left.\mathbb{P}_{(\infty)}^{1}\right)}\right)=\frac{1}{p^{2}}$.

(vii) $\epsilon\left(\mathbb{P}_{(\infty)}^{1},\left.[2]_{*} \overline{\mathbb{Q}}_{\ell}\right|_{\mathbb{P}_{(\infty)}^{1}},\left.d t\right|_{\left.\mathbb{P}_{(\infty)}^{1}\right)}\right)=-\frac{g(\chi, \psi)}{p^{4}}$.

(viii) $\epsilon\left(\mathbb{P}_{(\infty)}^{1},\left.j_{*}[2]_{*}\left(\mathcal{L}_{\psi}(a t) \otimes \mathcal{L}_{\chi}\right)\right|_{\mathbb{P}_{(\infty)}^{1}},\left.d t\right|_{\mathbb{P}_{(\infty)}^{1}}\right)=-\frac{g(\chi, \psi)}{p^{3}}\left(\frac{2 a}{p}\right)$.

(ix) $\epsilon\left(\mathbb{P}_{(\infty)}^{1},\left.j_{*}[2]_{*} \mathcal{L}_{\chi}\right|_{\mathbb{P}_{(\infty)}^{1}},\left.d t\right|_{\mathbb{P}_{(\infty)}^{1}}\right)=\frac{g(\chi, \psi)^{2}}{p^{4}}\left(\frac{-2}{p}\right)$.

(x) $\epsilon\left(\mathbb{P}_{(\infty)}^{1},\left.j_{*}[2]_{*} \mathcal{L}_{\psi}(a t)\right|_{\mathbb{P}_{(\infty)}^{1}},\left.d t\right|_{\mathbb{P}_{(\infty)}^{1}}\right)=-\frac{g(\chi, \psi)}{p^{3}}$.

Proof. Let $K_{\infty}$ be the completion of the field $k\left(\eta_{\infty}\right)$, let $\mathcal{O}_{\infty}$ be the ring of integers in $K_{\infty}$, and let $s=\frac{1}{t}$. Then $s$ is a uniformizer of $K_{\infty}$. Denote the inclusion $\eta_{\infty} \rightarrow \mathbb{P}_{(\infty)}^{1}$ also by $j$. Let $V$ be a $\overline{\mathbb{Q}}_{\ell^{-}}$sheaf of rank 1 on $\eta_{\infty}$, and let $\phi: K_{\infty}^{*} \rightarrow \overline{\mathbb{Q}}_{\ell}^{*}$ be the character corresponding to $V$ via the reciprocity law. The Artin conductor $a(\phi)$ of $\phi$ is defined to be the smallest integer $m$ such that $\left.\phi\right|_{1+s^{m} \mathcal{O}_{\infty}}=1$. For any nonzero meromorphic differential 1-form $\omega=f d s$ on $\mathbb{P}_{(\infty)}^{1}$, define the order $v_{\infty}(\omega)$ of $\omega$ to be the valuation $v_{\infty}(f)$ of $f$. By [12], 3.1.5.4 (v), we have

$$
\epsilon\left(\mathbb{P}_{(\infty)}^{1}, j_{*} V, \omega\right)= \begin{cases}\phi\left(s^{v_{\infty}(\omega)}\right) p^{v_{\infty}(\omega)} & \text { if }\left.\phi\right|_{\mathcal{O}_{\infty}^{*}}=1, \\ \int_{s^{-\left(a(\phi)+v_{\infty}(\omega)\right)} \mathcal{O}_{\infty}^{*}} \phi^{-1}(z) \psi\left(\operatorname{Res}_{\infty}(z \omega)\right) d z & \text { if }\left.\phi\right|_{\mathcal{O}_{\infty}^{*}} \neq 1,\end{cases}
$$

where $\operatorname{Res}_{\infty}$ denotes the residue of a meromorphic 1-form at $\infty$, and the integral is taken with respect to the Haar measure $d z$ on $K_{\infty}$ normalized by $\int_{\mathcal{O}_{\infty}} d z=1$.

Note that $d t=-\frac{d s}{s^{2}}$ has order -2 at $\infty$ and $d t^{2}=-\frac{2 d s}{s^{3}}$ has order -3 . Applying the first case of the above formula for the $\epsilon$-factor, we get (i) and (ii).

(iii) Taking $a=t=\frac{1}{s}$ and $b=z$ in the explicit reciprocity law in [18], XIV, §3, Proposition 8, we obtain that the character

$$
\chi^{\prime}: K_{\infty}^{*} \rightarrow \overline{\mathbb{Q}}_{l}^{*}
$$

corresponding to $\mathcal{L}_{\chi}$ is given by

$$
\chi^{\prime}(z)=\chi^{-1}\left(\left(\frac{\bar{c}}{p}\right)\right)
$$


where

$$
c=(-1)^{-v_{\infty}(z)} \frac{z^{-1}}{s^{-v_{\infty}(z)}}
$$

which is a unit in $\mathcal{O}_{\infty}, \bar{c}$ is the residue class of $c$ in $\mathcal{O}_{\infty} / s \mathcal{O}_{\infty} \cong \mathbb{F}_{p}$, and $\left(\frac{\bar{c}}{p}\right)$ is the Legendre symbol of $\bar{c}$. Note that our formula for $c$ is the reciprocal of the formula in [18] because the reciprocity map in [18 maps uniformizers in $K$ to arithmetic Frobenius elements in $\operatorname{Gal}\left(\bar{K}_{\infty} / K_{\infty}\right)^{\text {ab }}$, whereas the reciprocity map in [12] maps uniformizers in $K$ to geometric Frobenius elements. One can verify that $a\left(\chi^{\prime}\right)=1$. For any $z \in s \mathcal{O}_{\infty}^{*}$, write

$$
z=s\left(r_{0}+r_{1} s+\cdots\right)
$$

with $r_{i} \in \mathbb{F}_{p}$ and $r_{0} \neq 0$. We then have

$$
\begin{aligned}
\bar{c} & =-r_{0}^{-1}, \\
\operatorname{Res}_{\infty}(z d t) & =-r_{0} .
\end{aligned}
$$

So we have

$$
\begin{aligned}
\epsilon\left(\mathbb{P}_{(\infty)}^{1},\left.j_{*} \mathcal{L}_{\chi}\right|_{\left.\mathbb{P}_{(\infty)}^{1}\right)},\left.d t\right|_{\left.\mathbb{P}_{(\infty)}^{1}\right)}\right) & =\int_{s \mathcal{O}_{\infty}^{*}} \chi^{\prime-1}(z) \psi\left(\operatorname{Res}_{\infty}(z d t)\right) d z \\
& =\int_{s \mathcal{O}_{\infty}^{*}} \chi\left(\left(\frac{-r_{0}^{-1}}{p}\right)\right) \psi\left(-r_{0}\right) d z \\
& =\int_{s \mathcal{O}_{\infty}^{*}}\left(\frac{-r_{0}}{p}\right) \psi\left(-r_{0}\right) d z \\
& =\sum_{r_{0} \in \mathbb{F}_{p}^{*}} \int_{r_{0} s\left(1+s \mathcal{O}_{\infty}\right)}\left(\frac{-r_{0}}{p}\right) \psi\left(-r_{0}\right) d z \\
& =\sum_{r_{0} \in \mathbb{F}_{p}^{*}}\left(\frac{-r_{0}}{p}\right) \psi\left(-r_{0}\right) \int_{r_{0} s\left(1+s \mathcal{O}_{\infty}\right)} d z \\
& =\frac{1}{p^{2}} \sum_{r_{0} \in \mathbb{F}_{p}^{*}}\left(\frac{-r_{0}}{p}\right) \psi\left(-r_{0}\right) \\
& =\frac{g(\chi, \psi)}{p^{2}} .
\end{aligned}
$$

(iv) We can use the same method as in (iii), or use the formula [12], 3.1.5.5 to get

$$
\begin{aligned}
\epsilon\left(\mathbb{P}_{(\infty)}^{1},\left.j_{*} \mathcal{L}_{\chi}\right|_{\mathbb{P}_{(\infty)}^{1}},\left.d t^{2}\right|_{\left.\mathbb{P}_{(\infty)}^{1}\right)}\right) & =\epsilon\left(\mathbb{P}_{(\infty)}^{1},\left.j_{*} \mathcal{L}_{\chi}\right|_{\left.\mathbb{P}_{(\infty)}^{1}\right)},\left.2 t d t\right|_{\mathbb{P}_{(\infty)}^{1}}\right) \\
& =\chi^{\prime}\left(\frac{2}{s}\right) p^{v_{\infty}\left(\frac{2}{s}\right)} \epsilon\left(\mathbb{P}_{(\infty)}^{1},\left.j_{*} \mathcal{L}_{\chi}\right|_{\left.\mathbb{P}_{(\infty)}^{1}\right)},\left.d t\right|_{\mathbb{P}_{(\infty)}^{1}}\right) \\
& =\left(\frac{-2}{p}\right) \cdot \frac{1}{p} \cdot \epsilon\left(\mathbb{P}_{(\infty)}^{1},\left.j_{*} \mathcal{L}_{\chi}\right|_{\left.\mathbb{P}_{(\infty)}^{1}\right)},\left.d t\right|_{\mathbb{P}_{(\infty)}^{1}}\right) \\
& =-\frac{g(\chi, \psi)}{p^{3}}\left(\frac{-2}{p}\right) .
\end{aligned}
$$

(v) Taking $a$ to be $a t=\frac{a}{s}$ and $b=z$ in the explicit reciprocity law in [18], XIV, $\S 5$, Proposition 15 , we see that the character

$$
K_{\infty}^{*} \rightarrow \overline{\mathbb{Q}}_{l}^{*}
$$


corresponding to $\mathcal{L}_{\chi}(a t)$ is

$$
z \mapsto \psi^{-1}\left(-\operatorname{Res}_{\infty}\left(\frac{a}{s} \cdot \frac{d z}{z}\right)\right) .
$$

(We add the negative sign to the formula in [18] since the reciprocity map in [18] is different from the one used in [12.) So the character

$$
\phi: K_{\infty}^{*} \rightarrow \overline{\mathbb{Q}}_{l}^{*}
$$

corresponding to $\mathcal{L}_{\chi}(a t) \otimes \mathcal{L}_{\chi}$ is given by

$$
\phi(z)=\psi^{-1}\left(-\operatorname{Res}_{\infty}\left(\frac{a}{s} \cdot \frac{d z}{z}\right)\right) \chi^{-1}\left(\left(\frac{\bar{c}}{p}\right)\right),
$$

where $c=(-1)^{-v_{\infty}(z)} \frac{z^{-1}}{s^{-v_{\infty}(z)}}$. One can verify that $a(\phi)=2$. For any $z \in s \mathcal{O}_{\infty}^{*}$, write

$$
z=s\left(r_{0}+r_{1} s+\cdots\right)
$$

with $r_{i} \in \mathbb{F}_{p}$ and $r_{0} \neq 0$. We then have

$$
\begin{aligned}
\operatorname{Res}_{\infty}\left(\frac{a}{s} \cdot \frac{d z}{z}\right) & =\frac{a r_{1}}{r_{0}} \\
\bar{c} & =-r_{0}^{-1}, \\
\operatorname{Res}_{\infty}\left(z d t^{2}\right) & =-2 r_{1} .
\end{aligned}
$$

So we have

$$
\begin{aligned}
& \epsilon\left(\mathbb{P}_{(\infty)}^{1},\left.j_{*} \mathcal{L}_{\chi}\right|_{\left.\mathbb{P}_{(\infty)}^{1}\right)},\left.d t^{2}\right|_{\left.\mathbb{P}_{(\infty)}^{1}\right)}\right) \\
= & \int_{s \mathcal{O}_{\infty}^{*}} \phi^{-1}(z) \psi\left(\operatorname{Res}_{\infty}\left(z d t^{2}\right)\right) d z \\
= & \int_{s \mathcal{O}_{\infty}^{*}} \psi\left(-\frac{a r_{1}}{r_{0}}\right) \chi\left(\left(\frac{-r_{0}^{-1}}{p}\right)\right) \psi\left(-2 r_{1}\right) d z \\
= & \int_{s \mathcal{O}_{\infty}^{*}}\left(\frac{-r_{0}}{p}\right) \psi\left(-r_{1}\left(\frac{a}{r_{0}}+2\right)\right) d z \\
= & \sum_{r_{0}, r_{1} \in \mathbb{F}_{p}, r_{0} \neq 0} \int_{s\left(r_{0}+r_{1} s\right)\left(1+s^{2} \mathcal{O}_{\infty}\right)}\left(\frac{-r_{0}}{p}\right) \psi\left(-r_{1}\left(\frac{a}{r_{0}}+2\right)\right) d z \\
= & \sum_{r_{0}, r_{1} \in \mathbb{F}_{p}, r_{0} \neq 0}\left(\frac{-r_{0}}{p}\right) \psi\left(-r_{1}\left(\frac{a}{r_{0}}+2\right)\right) \int_{s\left(r_{0}+r_{1} s\right)\left(1+s^{2} \mathcal{O}_{\infty}\right)} d z \\
= & \frac{1}{p^{3}} \sum_{r_{0}, r_{1} \in \mathbb{F}_{p}, r_{0} \neq 0}\left(\frac{-r_{0}}{p}\right) \psi\left(-r_{1}\left(\frac{a}{r_{0}}+2\right)\right) \\
= & \frac{1}{p^{3}} \sum_{r_{0} \in \mathbb{F}_{p}^{*}}\left(\frac{-r_{0}}{p}\right) \sum_{r_{1} \in \mathbb{F}_{p}} \psi\left(-r_{1}\left(\frac{a}{r_{0}}+2\right)\right) \\
= & \frac{1}{p^{3}} \cdot\left(\frac{-\frac{a}{2}}{p}\right) \cdot p \\
= & \frac{1}{p^{2}}\left(\frac{2 a}{p}\right) \cdot
\end{aligned}
$$

We omit the proof of (vi), which is similar to the proof of (v). 
(vii) We have $[2]_{*} \overline{\mathbb{Q}}_{\ell} \cong \overline{\mathbb{Q}}_{\ell} \oplus j_{*} \mathcal{L}_{\chi}$. So

$$
\epsilon\left(\mathbb{P}_{(\infty)}^{1},\left.[2]_{*} \overline{\mathbb{Q}}_{\ell}\right|_{\mathbb{P}_{(\infty)}^{1}},\left.d t\right|_{\mathbb{P}_{(\infty)}^{1}}\right)=\epsilon\left(\mathbb{P}_{(\infty)}^{1},\left.\overline{\mathbb{Q}}_{\ell}\right|_{\mathbb{P}_{(\infty)}^{1}},\left.d t\right|_{\mathbb{P}_{(\infty)}^{1}}\right) \epsilon\left(\mathbb{P}_{(\infty)}^{1},\left.j_{*} \mathcal{L}_{\chi}\right|_{\mathbb{P}_{(\infty)}^{1}},\left.d t\right|_{\left.\mathbb{P}_{(\infty)}^{1}\right)}\right) .
$$

We then use (i) and (iii).

(viii) We can define $\epsilon$-factors for virtual sheaves on $\mathbb{P}_{(\infty)}^{1}$. By [12, 3.1.5.4 (iv), we have

$$
\begin{aligned}
& \epsilon\left(\mathbb{P}_{(\infty)}^{1},\left.[2]_{*}\left(\left[j_{*}\left(\mathcal{L}_{\psi}(a t) \otimes \mathcal{L}_{\chi}\right)\right]-\left[\overline{\mathbb{Q}}_{\ell}\right]\right)\right|_{\mathbb{P}_{(\infty)}^{1}},\left.d t\right|_{\mathbb{P}_{(\infty)}^{1}}\right) \\
& \quad=\epsilon\left(\mathbb{P}_{(\infty)}^{1},\left.\left(\left[j_{*}\left(\mathcal{L}_{\psi}(a t) \otimes \mathcal{L}_{\chi}\right)\right]-\left[\overline{\mathbb{Q}}_{\ell}\right]\right)\right|_{\mathbb{P}_{(\infty)}^{1}},\left.d t^{2}\right|_{\mathbb{P}_{(\infty)}^{1}}\right) .
\end{aligned}
$$

Hence

$$
\begin{aligned}
& \epsilon\left(\mathbb{P}_{(\infty)}^{1},\left.j_{*}[2]_{*}\left(\mathcal{L}_{\psi}(a t) \otimes \mathcal{L}_{\chi}\right)\right|_{\mathbb{P}_{(\infty)}^{1}},\left.d t\right|_{\left.\mathbb{P}_{(\infty)}^{1}\right)}\right) \\
= & \frac{\epsilon\left(\mathbb{P}_{(\infty)}^{1},\left.j_{*}\left(\mathcal{L}_{\psi}(a t) \otimes \mathcal{L}_{\chi}\right)\right|_{\mathbb{P}_{(\infty)}^{1}},\left.d t^{2}\right|_{\left.\mathbb{P}_{(\infty)}^{1}\right)}\right)}{\epsilon\left(\mathbb{P}_{(\infty)}^{1}, \overline{\mathbb{Q}}_{\ell},\left.d t^{2}\right|_{\mathbb{P}_{(\infty)}^{1}}\right)} \epsilon\left(\mathbb{P}_{(\infty)}^{1},[2]_{*} \overline{\mathbb{Q}}_{\ell},\left.d t\right|_{\mathbb{P}_{(\infty)}^{1}}\right) .
\end{aligned}
$$

We then apply the formulas (ii), (v), and (vii).

We omit the proofs of (ix) and (x), which are similar to the proof of (viii).

Lemma 2.6. We have

$$
\begin{aligned}
& \epsilon\left(\mathbb{P}_{(\infty)}^{1},\left.j_{*}\left(\mathcal{L}_{\chi^{r}} \otimes \mathcal{L}_{\theta_{0}^{2 r} \theta_{1}^{r}}\right)\right|_{\mathbb{P}_{(\infty)}^{1}},\left.d t\right|_{\mathbb{P}_{(\infty)}^{1}}\right) \\
& = \begin{cases}\frac{g(\chi, \psi)^{-4 r}}{p^{2}} & \text { if } r \text { is even, } \\
-\frac{g(\chi, \psi)^{-2 r+1}}{p^{2}}\left(\frac{-1}{p}\right) & \text { if } r \text { is odd, }\end{cases} \\
& \epsilon\left(\mathbb{P}_{(\infty)}^{1},\left.j_{*}\left([2]_{*} \mathcal{L}_{\psi}((4 i-4 r) t) \otimes \mathcal{L}_{\theta_{0}^{2 r} \theta_{1}^{i}}\right)\right|_{\mathbb{P}_{(\infty)}^{1}},\left.d t\right|_{\mathbb{P}_{(\infty)}^{1}}\right) \\
& = \begin{cases}-\frac{g(\chi, \psi)^{-6 r+1}}{p^{4}}\left(\frac{-1}{p}\right)^{i} & \text { if } p \mid i-r, \\
-\frac{g(\chi, \psi)^{-2 r+1}}{p^{3}}\left(\frac{-1}{p}\right)^{i} & \text { if } p \not i-r,\end{cases} \\
& \left.\left.\epsilon\left(\mathbb{P}_{(\infty)}^{1}, j_{*}\left([2]_{*} \mathcal{L}_{\psi}((4 i-4 r-2) t) \otimes \mathcal{L}_{\chi}\right) \otimes \mathcal{L}_{\theta_{0}^{2 r+1} \theta_{1}^{i+1}}\right)\right|_{\mathbb{P}_{(\infty)}^{1}},\left.d t\right|_{\mathbb{P}_{(\infty)}^{1}}\right) \\
& = \begin{cases}\frac{g(\chi, \psi)^{-4 r}}{p^{4}}\left(\frac{-2}{p}\right) & \text { if } p \mid 2 i-2 r-1, \\
-\frac{g(\chi, \psi)^{-2 r}}{p^{3}}\left(\frac{(-1)^{i+1}(2 i-2 r-1)}{p}\right) & \text { if } p \nmid 2 i-2 r-1 .\end{cases}
\end{aligned}
$$

Proof. Let $F_{\infty}$ be the geometric Frobenius element at $\infty$. We have

$$
\theta_{0}\left(F_{\infty}\right)=g(\chi, \psi), \theta_{1}\left(F_{\infty}\right)=\left(\frac{-1}{p}\right) .
$$

Using the notation in [12, 3.1.5.1, we have

$$
\begin{aligned}
a\left(\mathbb{P}_{(\infty)}^{1}, \overline{\mathbb{Q}}_{\ell},\left.d t\right|_{\left.\mathbb{P}_{(\infty)}^{1}\right)}\right) & =-2, \\
a\left(\mathbb{P}_{(\infty)}^{1},\left.j_{*} \mathcal{L}_{\chi}\right|_{\mathbb{P}_{(\infty)}^{1}},\left.d t\right|_{\mathbb{P}_{(\infty)}^{1}}\right) & =-1, \\
a\left(\mathbb{P}_{(\infty)}^{1},[2]_{*} \overline{\mathbb{Q}}_{\ell},\left.d t\right|_{\mathbb{P}_{(\infty)}^{1}}\right) & =-3, \\
a\left(\mathbb{P}_{(\infty)}^{1},\left.j_{*}[2]_{*} \mathcal{L}_{\psi}(a t)\right|_{\mathbb{P}_{(\infty)}^{1}},\left.d t\right|_{\mathbb{P}_{(\infty)}^{1}}\right) & =-1\left(a \in \mathbb{F}_{p}^{*}\right), \\
a\left(\mathbb{P}_{(\infty)}^{1},\left.j_{*}[2]_{*} \mathcal{L}_{\chi}\right|_{\mathbb{P}_{(\infty)}^{1}},\left.d t\right|_{\mathbb{P}_{(\infty)}^{1}}\right) & =-2, \\
a\left(\mathbb{P}_{(\infty)}^{1},\left.j_{*}[2]_{*}\left(\mathcal{L}_{\psi}(a t) \otimes \mathcal{L}_{\chi}\right)\right|_{\mathbb{P}_{(\infty)}^{1}},\left.d t\right|_{\mathbb{P}_{(\infty)}^{1}}\right) & =-1\left(a \in \mathbb{F}_{p}^{*}\right) .
\end{aligned}
$$


So by [12], 3.1.5.6, we have

$$
\begin{aligned}
& \epsilon\left(\mathbb{P}_{(\infty)}^{1},\left.j_{*}\left(\mathcal{L}_{\chi^{r}} \otimes \mathcal{L}_{\theta_{0}^{2 r} \theta_{1}^{r}}\right)\right|_{\mathbb{P}_{(\infty)}^{1}},\left.d t\right|_{\mathbb{P}_{(\infty)}^{1}}\right) \\
& = \begin{cases}\left(\left(\theta_{0}^{2 r} \theta_{1}^{r}\right)\left(F_{\infty}\right)\right)^{-2} \epsilon\left(\mathbb{P}_{(\infty)}^{1}, \overline{\mathbb{Q}}_{\ell},\left.d\right|_{\mathbb{P}_{(\infty)}^{1}}\right) & \text { if } r \text { is even, } \\
\left(\left(\theta_{0}^{2 r} \theta_{1}^{r}\right)\left(F_{\infty}\right)\right)^{-1} \epsilon\left(\mathbb{P}_{(\infty)}^{1},\left.j_{*} \mathcal{L}_{\chi}\right|_{\left.\mathbb{P}_{(\infty)}^{1}\right)},\left.d t\right|_{\mathbb{P}_{(\infty)}^{1}}\right) & \text { if } r \text { is odd, }\end{cases} \\
& \epsilon\left(\mathbb{P}_{(\infty)}^{1},\left.j_{*}\left([2]_{*} \mathcal{L}_{\psi}((4 i-4 r) t) \otimes \mathcal{L}_{\theta_{0}^{2 r} \theta_{1}^{i}}\right)\right|_{\mathbb{P}_{(\infty)}^{1}},\left.d t\right|_{\mathbb{P}_{(\infty)}^{1}}\right) \\
& = \begin{cases}\left(\left(\theta_{0}^{2 r} \theta_{1}^{i}\right)\left(F_{\infty}\right)\right)^{-3} \epsilon\left(\mathbb{P}_{(\infty)}^{1},[2]_{*} \overline{\mathbb{Q}}_{\ell},\left.d t\right|_{\left.\mathbb{P}_{(\infty)}^{1}\right)}\right) & \text { if } p \mid i-r, \\
\left(\left(\theta_{0}^{2 r} \theta_{1}^{i}\right)\left(F_{\infty}\right)\right)^{-1} \epsilon\left(\mathbb{P}_{(\infty)}^{1},\left.j_{*}[2]_{*} \mathcal{L}_{\psi}((4 i-4 r) t)\right|_{\mathbb{P}_{(\infty)}^{1}},\left.d t\right|_{\left.\mathbb{P}_{(\infty)}^{1}\right)}\right) & \text { if } p \nmid i-r,\end{cases} \\
& \epsilon\left(\mathbb{P}_{(\infty)}^{1},\left.j_{*}\left([2]_{*}\left(\mathcal{L}_{\psi}((4 i-4 r-2) t) \otimes \mathcal{L}_{\chi}\right) \otimes \mathcal{L}_{\theta_{0}^{2 r+1} \theta_{1}^{i+1}}\right)\right|_{\mathbb{P}_{(\infty)}^{1}},\left.d t\right|_{\mathbb{P}_{(\infty)}^{1}}\right) \\
& =\left\{\begin{array}{r}
\left(\left(\theta_{0}^{2 r+1} \theta_{1}^{i+1}\right)\left(F_{\infty}\right)\right)^{-2} \epsilon\left(\mathbb{P}_{(\infty)}^{1},\left.j_{*}[2]_{*} \mathcal{L}_{\chi}\right|_{\mathbb{P}_{(\infty)}^{1}},\left.d t\right|_{\left.\mathbb{P}_{(\infty)}^{1}\right)} \text { if } p \mid 2 i-2 r-1,\right. \\
\left(\left(\theta_{0}^{2 r+1} \theta_{1}^{i+1}\right)\left(F_{\infty}\right)\right)^{-1} \epsilon\left(\mathbb{P}_{(\infty)}^{1},\left.j_{*}[2]_{*}\left(\mathcal{L}_{\psi}((4 i-4 r-2) t) \otimes \mathcal{L}_{\chi}\right)\right|_{\mathbb{P}_{(\infty)}^{1}},\left.d t\right|_{\mathbb{P}_{(\infty)}^{1}}\right) \\
\text { if } p \nmid 2 i-2 r-1 .
\end{array}\right.
\end{aligned}
$$

We then apply the formulas in Lemma 2.5.

The following is Proposition 0.4 in the Introduction.

Proposition 2.7. $\epsilon\left(\mathbb{P}_{(\infty)}^{1},\left.j_{*}\left(\operatorname{Sym}^{k}\left(\mathrm{Kl}_{2}\right)\right)\right|_{\left.\mathbb{P}_{(\infty)}^{1}\right)},\left.d t\right|_{\left.\mathbb{P}_{(\infty)}^{1}\right)}\right)$ equals

$$
p^{-(k+1)\left(\frac{k+8}{4}+\left[\frac{k}{2 p}\right]\right)}
$$

if $k=2 r$ for an even $r$,

$$
p^{-(k+1)\left(\frac{k+6}{4}+\left[\frac{k}{2 p}\right]\right)}
$$

if $k=2 r$ for an odd $r$, and

$$
(-1)^{\frac{k+1}{2}+\left[\frac{k}{p}\right]-\left[\frac{k}{2 p}\right]} p^{-\frac{k+1}{2}\left(\frac{k+5}{2}+\left[\frac{k}{p}\right]-\left[\frac{k}{2 p}\right]\right)}\left(\frac{-2}{p}\right)^{\left[\frac{k}{p}\right]-\left[\frac{k}{2 p}\right]} \prod_{\substack{j \in\left\{0,1, \ldots,\left[\frac{k}{2}\right]\right\}, p \nmid 2 j+1}}\left(\frac{(-1)^{j}(2 j+1)}{p}\right)
$$

if $k=2 r+1$.

Proof. By Lemmas 2.4 and 2.6, $\epsilon\left(\mathbb{P}_{(\infty)}^{1},\left.j_{*}\left(\operatorname{Sym}^{k}\left(\mathrm{Kl}_{2}\right)\right)\right|_{\mathbb{P}_{(\infty)}^{1}},\left.d t\right|_{\mathbb{P}_{(\infty)}^{1}}\right)$ equals

$$
\begin{aligned}
\frac{g(\chi, \psi)^{-4 r}}{p^{2}} \prod_{i \in\{0, \ldots, r-1\}, p \mid i-r} & \left(-\frac{g(\chi, \psi)^{-6 r+1}}{p^{4}}\left(\frac{-1}{p}\right)^{i}\right) \\
& \times \prod_{i \in\{0, \ldots, r-1\}, p \nmid i-r}\left(-\frac{g(\chi, \psi)^{-2 r+1}}{p^{3}}\left(\frac{-1}{p}\right)^{i}\right)
\end{aligned}
$$

if $k=2 r$ for an even $r$,

$$
\begin{aligned}
-\frac{g(\chi, \psi)^{-2 r+1}}{p^{2}}\left(\frac{-1}{p}\right) \prod_{i \in\{0, \ldots, r-1\}, p \mid i-r} & \left(-\frac{g(\chi, \psi)^{-6 r+1}}{p^{4}}\left(\frac{-1}{p}\right)^{i}\right) \\
\times & \prod_{i \in\{0, \ldots, r-1\}, p \nmid i-r}\left(-\frac{g(\chi, \psi)^{-2 r+1}}{p^{3}}\left(\frac{-1}{p}\right)^{i}\right)
\end{aligned}
$$


if $k=2 r$ for an odd $r$, and

$$
\begin{aligned}
\prod_{i \in\{0, \ldots, r\}, p \mid 2 i-2 r-1} & \left(\frac{g(\chi, \psi)^{-4 r}}{p^{4}}\left(\frac{-2}{p}\right)\right) \\
\times & \prod_{i \in\{0, \ldots, r\}, p \nmid 2 i-2 r-1}\left(-\frac{g(\chi, \psi)^{-2 r}}{p^{3}}\left(\frac{(-1)^{i+1}(2 i-2 r-1)}{p}\right)\right)
\end{aligned}
$$

if $k=2 r+1$. Let's simplify the above expressions. Recall that $g(\chi, \psi)^{2}=p\left(\frac{-1}{p}\right)$. If $k=2 r$ with $r$ even, we have

$$
\begin{aligned}
& \epsilon\left(\mathbb{P}_{(\infty)}^{1},\left.j_{*}\left(\operatorname{Sym}^{k}\left(\mathrm{Kl}_{2}\right)\right)\right|_{\left.\mathbb{P}_{(\infty)}^{1}\right)},\left.d t\right|_{\left.\mathbb{P}_{(\infty)}^{1}\right)}\right) \\
&= \frac{g(\chi, \psi)^{-4 r}}{p^{2}} \prod_{i \in\{0, \ldots, r-1\}, p \mid i-r}\left(-\frac{g(\chi, \psi)^{-6 r+1}}{p^{4}}\left(\frac{-1}{p}\right)^{i}\right) \\
& \times \prod_{i \in\{0, \ldots, r-1\}, p \nmid i-r}\left(-\frac{g(\chi, \psi)^{-2 r+1}}{p^{3}}\left(\frac{-1}{p}\right)^{i}\right) \\
&=\frac{g(\chi, \psi)^{-4 r}}{p^{2}} \prod_{i \in\{0, \ldots, r-1\}, p \mid i-r} \frac{g(\chi, \psi)^{-4 r}}{p} \prod_{i \in\{0, \ldots, r-1\}}\left(-\frac{g(\chi, \psi)^{-2 r+1}}{p^{3}}\left(\frac{-1}{p}\right)^{i}\right) \\
&=\frac{g(\chi, \psi)^{-4 r}}{p^{2}}\left(\frac{g(\chi, \psi)^{-4 r}}{p}\right)^{\left[\frac{r}{p}\right]}\left(-\frac{g(\chi, \psi)^{-2 r+1}}{p^{3}}\right)^{r}\left(\frac{-1}{p}\right)^{\frac{r(r-1)}{2}} \\
&=\frac{p^{-2 r}}{p^{2}}\left(\frac{p^{-2 r}}{p}\right)^{\left[\frac{r}{p}\right]} \frac{\left(p\left(\frac{-1}{p}\right)\right)^{\frac{r(-2 r+1)}{2}}}{p^{3 r}}\left(\frac{-1}{p}\right)^{\frac{r(r-1)}{2}} \\
&=p^{-r^{2}-\frac{9}{2} r-2-(2 r+1)\left[\frac{r}{p}\right]\left(\frac{-1}{p}\right)^{-\frac{r^{2}}{2}}} \\
&=p^{-(k+1)\left(\frac{k+8}{4}+\left[\frac{k}{2 p}\right]\right)} .
\end{aligned}
$$

If $k=2 r$ with $r$ odd, we have

$$
\begin{aligned}
& \epsilon\left(\mathbb{P}_{(\infty)}^{1},\left.j_{*}\left(\operatorname{Sym}^{k}\left(\mathrm{Kl}_{2}\right)\right)\right|_{\left.\mathbb{P}_{(\infty)}^{1}\right)},\left.d t\right|_{\mathbb{P}_{(\infty)}^{1}}\right) \\
& =-\frac{g(\chi, \psi)^{-2 r+1}}{p^{2}}\left(\frac{-1}{p}\right) \prod_{i \in\{0, \ldots, r-1\}, p \mid i-r}\left(-\frac{g(\chi, \psi)^{-6 r+1}}{p^{4}}\left(\frac{-1}{p}\right)^{i}\right) \\
& \times \prod_{i \in\{0, \ldots, r-1\}, p \nmid i-r}\left(-\frac{g(\chi, \psi)^{-2 r+1}}{p^{3}}\left(\frac{-1}{p}\right)^{i}\right) \\
& =-\frac{g(\chi, \psi)^{-2 r+1}}{p^{2}}\left(\frac{-1}{p}\right) \prod_{i \in\{0, \ldots, r-1\}, p \mid i-r}\left(\frac{g(\chi, \psi)^{-4 r}}{p}\right) \\
& \times \prod_{i \in\{0, \ldots, r-1\}}\left(-\frac{g(\chi, \psi)^{-2 r+1}}{p^{3}}\left(\frac{-1}{p}\right)^{i}\right) \\
& =-\frac{g(\chi, \psi)^{-2 r+1}}{p^{2}}\left(\frac{-1}{p}\right)\left(\frac{g(\chi, \psi)^{-4 r}}{p}\right)^{\left[\frac{r}{p}\right]}\left(-\frac{g(\chi, \psi)^{-2 r+1}}{p^{3}}\right)^{r}\left(\frac{-1}{p}\right)^{\frac{r(r-1)}{2}}
\end{aligned}
$$




$$
\begin{aligned}
& =\frac{g(\chi, \psi)^{(-2 r+1)(r+1)}}{p^{3 r+2}}\left(\frac{-1}{p}\right)^{1+\frac{r(r-1)}{2}}\left(\frac{g(\chi, \psi)^{-4 r}}{p}\right)^{\left[\frac{r}{p}\right]} \\
& =\frac{\left(p\left(\frac{-1}{p}\right)\right)^{\frac{(-2 r+1)(r+1)}{2}}}{p^{3 r+2}}\left(\frac{-1}{p}\right)^{1+\frac{r(r-1)}{2}}\left(\frac{p^{-2 r}}{p}\right)^{\left[\frac{r}{p}\right]} \\
& =p^{-r^{2}-\frac{7}{2} r-\frac{3}{2}-(2 r+1)\left[\frac{r}{p}\right]}\left(\frac{-1}{p}\right)^{-\frac{(r-1)(r+3)}{2}} \\
& =p^{-(k+1)\left(\frac{k+6}{4}+\left[\frac{k}{2 p}\right]\right)} .
\end{aligned}
$$

If $k=2 r+1$ is odd, we have

$$
\begin{aligned}
& \epsilon\left(\mathbb{P}_{(\infty)}^{1},\left.j_{*}\left(\operatorname{Sym}^{k}\left(\mathrm{Kl}_{2}\right)\right)\right|_{\mathbb{P}_{(\infty)}^{1}},\left.d t\right|_{\mathbb{P}_{(\infty)}^{1}}\right) \\
& =\prod_{i \in\{0, \ldots, r\}, p \mid 2 i-2 r-1}\left(\frac{g(\chi, \psi)^{-4 r}}{p^{4}}\left(\frac{-2}{p}\right)\right) \\
& \times \prod_{i \in\{0, \ldots, r\}, p \nmid 2 i-2 r-1}\left(-\frac{g(\chi, \psi)^{-2 r}}{p^{3}}\left(\frac{(-1)^{i+1}(2 i-2 r-1)}{p}\right)\right) \\
& =\left(-\frac{g(\chi, \psi)^{-2 r}}{p^{3}}\right)^{r+1}\left(-\frac{g(\chi, \psi)^{-2 r}}{p}\left(\frac{-2}{p}\right)\right)^{\left[\frac{k}{p}\right]-\left[\frac{k}{2 p}\right]} \\
& \times \prod_{i \in\{0, \ldots, r\}, p \nmid 2 i-2 r-1}\left(\frac{(-1)^{i+1}(2 i-2 r-1)}{p}\right) \\
& =\left(-p^{-3-r}\left(\frac{-1}{p}\right)^{-r}\right)^{r+1}\left(-p^{-1-r}\left(\frac{-1}{p}\right)^{-r}\left(\frac{-2}{p}\right)\right)^{\left[\frac{k}{p}\right]-\left[\frac{k}{2 p}\right]} \\
& \times \prod_{j \in\{0, \ldots, r\}, p \nmid 2 j+1}\left(\frac{(-1)^{r-j}(2 j+1)}{p}\right) \\
& =(-1)^{r+1+\left[\frac{k}{p}\right]-\left[\frac{k}{2 p}\right]} p^{-(r+1)\left(r+3+\left[\frac{k}{p}\right]-\left[\frac{k}{2 p}\right]\right)}\left(\frac{-2}{p}\right)^{\left[\frac{k}{p}\right]-\left[\frac{k}{2 p}\right]}\left(\frac{-1}{p}\right)^{-r(r+1)-r\left(\left[\frac{k}{p}\right]-\left[\frac{k}{2 p}\right]\right)} \\
& \times \prod_{j \in\{0, \ldots, r\}, p \nmid 2 j+1}\left(\frac{(-1)^{r-j}(2 j+1)}{p}\right) \\
& =(-1)^{\frac{k+1}{2}+\left[\frac{k}{p}\right]-\left[\frac{k}{2 p}\right]} p^{-\frac{k+1}{2}\left(\frac{k+5}{2}+\left[\frac{k}{p}\right]-\left[\frac{k}{2 p}\right]\right)}\left(\frac{-2}{p}\right)^{\left[\frac{k}{p}\right]-\left[\frac{k}{2 p}\right]} \\
& \times \prod_{j \in\left\{0, \ldots,\left[\frac{k}{2}\right]\right\}, p \nmid 2 j+1}\left(\frac{(-1)^{j}(2 j+1)}{p}\right) .
\end{aligned}
$$

\section{REFERENCES}

[1] M. Artin, A. Grothendieck, and J.-L. Verdier, Théorie des topos et cohomologie étale des schémas (SGA 4), Lecture Notes in Math. 269, 270, 305, Springer-Verlag (1972-1973). MR0354652(50:7130) MR0354653(50:7131) MR0354654(50:7132)

[2] H. T. Choi and R. J. Evans, Congruences for sums of powers of Kloosterman sums, Inter. J. Number Theory, 3(2007), 105-117. MR2310495 (2008d:11090) 
[3] P. Deligne, Applications de la Formule des Traces aux Sommes Trigonométriques, in Cohomologie Étale (SGA $4 \frac{1}{2}$ ), 168-232, Lecture Notes in Math. 569, Springer-Verlag, 1977. MR0463174 (57:3132)

[4] R. J. Evans, Seventh power moments of Kloosterman sums, Israel J. Math., to appear.

[5] R. J. Evans, Letter to N. Katz, Nov. 2005.

[6] L. Fu, Calculation of $\ell$-adic local Fourier transformations, arXiv: 0702436 [math.AG] (2007).

[7] L. Fu and D. Wan, Trivial factors for $L$-functions of symmetric products of Kloosterman sheaves, Finite Fields and Appl. 14 (2008), 549-570. MR2401995 (2009c:11135)

[8] L. Fu and D. Wan, $L$-functions for symmetric products of Kloosterman sums, J. Reine Angew. Math. 589 (2005), 79-103. MR2194679 (2006k:11159)

[9] L. Fu and D. Wan, $L$-functions of symmetric products of the Kloosterman sheaf over $\mathbb{Z}$, Math. Ann. 342 (2008), 387-404. MR 2425148 (2009i:14022)

[10] K. Hulek, J. Spandaw, B. van Geemen and D. van Straten, The modularity of the Barth-Nieto quintic and its relative, Adv. Geom., 1(2001), no.3, 263-289. MR.1874236 (2003b:11058)

[11] N. Katz, Gauss sums, Kloosterman sums, and monodromy groups, Princeton University Press, 1988. MR 955052 (91a:11028)

[12] G. Laumon, Transformation de Fourier, constantes d'équations fonctionnelles, et conjecture de Weil, Publ. Math. IHES 65 (1987), 131-210. MR908218 (88g:14019)

[13] M. Moisio, The moments of Kloosterman sums and the weight distribution of a Zetterbergtype binary cyclic code, IEEE, Trans. Inform. Theory, 53(2007), 843-847. MR2302793 (2008c:11111)

[14] M. Moisio, On the moments of Kloosterman sums and fibre products of Kloosterman curves, Finite Fields Appl., 14(2008), 515-531. MR2401992 (2009e:11226)

[15] C. Peters, J. Top and M. van der Vlugt, The Hasse zeta function of a K3 surface related to the number of words of weight 5 in the Melas codes, J. Reine Angew. Math. 432(1992), 151-176. MR.1184764(94d:11044)

[16] P. Robba, Symmetric powers of p-adic Bessel functions, J. Reine Angew. Math., 366(1986), 194-220. MR833018 (87f:12016)

[17] J.-P. Serre, Linear representations of finite groups, Springer-Verlag, 1977. MR 0450380 $(56: 8675)$

[18] J.-P. Serre, Local fields, Springer-Verlag, 1979. MR554237 (82e:12016)

[19] D. Wan, Dwork's conjecture on unit root zeta functions, Ann. of Math. (2), 150(1999), 867927. MR.1740990 (2001a:11108)

Institute of Mathematics, Nankai University, Tianjin, People's Republic of China

E-mail address: leifu@nankai.edu.cn

Department of Mathematics, University of California, Irvine, California 92697

E-mail address: dwan@math.uci.edu 